\title{
ON SOME GENERALIZATIONS OF JACOBI'S RESIDUE FORMULA
}

\author{
By AlEKos VIDRAS AND ALAIN YGER
}

ABSTRACT. - Using Bochner-Martinelli type residual currents we prove some generalizations of Jacobi’s residue formula, which allow proper polynomial maps to have 'common zeroes at infinity', in projective or toric situations. (C) 2001 Éditions scientifiques et médicales Elsevier SAS

RÉSUMÉ. $-\mathrm{Si} \mathcal{D}_{1}, \ldots, \mathcal{D}_{n}$ sont $n$ diviseurs s'intersectant proprement sur une variété analytique complexe compacte $\mathcal{X}$ de dimension $n$ et si $\omega$ est une forme méromorphe sur $\mathcal{X}$ de lieu polaire inclus dans l'union des supports des $\mathcal{D}_{j}$, il résulte d'un théorème de Griffiths que la somme des résidus de Grothendieck de $\omega$ en tous les points de $\left|\mathcal{D}_{1}\right| \cap \cdots \cap\left|\mathcal{D}_{n}\right|$ est nulle. Les formules de Bochner-Martinelli permettent d'étendre ce résultat (dans les cadres projectif et torique) sous des hypothèses d'intersection propre hors de la variété à l'infini. Des applications géométriques (du type Cayley-Bacharach) ou algébriques (effectivité de la division ou de l'identité de Bézout) illustrent les énoncés. (C) 2001 Éditions scientifiques et médicales Elsevier SAS

\section{Introduction}

One of the classical results in the one complex variable residue theory is the following: for every polynomial map $P: \mathbb{C} \rightarrow \mathbb{C}$, the total sum of residues of the form $Q \mathrm{~d} \zeta / P$ (where $Q \in \mathbb{C}[X])$ at the zeroes of $P$ equals the residue at infinity of the rational function $Q / P$ with the opposite sign.

Some multidimensional analogues of this statement are treated in the present note. Consider a polynomial map

$$
P=\left(P_{1}, \ldots, P_{n}\right): \mathbb{C}^{n} \longrightarrow \mathbb{C}^{n}
$$

and assume that $\mathbb{C}^{n}$ is imbedded into the complex projective space $\mathbb{P}^{n}$. Let ${ }^{\mathrm{h}} P_{1}, \ldots,{ }^{\mathrm{h}} P_{n}$ be the homogenizations of the $P_{j}, j=1, \ldots, n$, that is the homogeneous polynomials in $n+1$ variables

$$
{ }^{\mathrm{h}} P_{j}\left(X_{0}, X_{1}, \ldots, X_{n}\right)=X_{0}^{\operatorname{deg} P_{j}} P_{j}\left(\frac{X_{1}}{X_{0}}, \ldots, \frac{X_{n}}{X_{0}}\right) .
$$

Let us impose the Jacobi condition, that is:

$$
\begin{aligned}
& \text { the homogeneous parts of highest degree in } P_{j}\left(X_{1}, \ldots, X_{n}\right) \text {, for } \\
& j=1, \ldots, n \text {, do not have common zeroes in } \mathbb{C}^{n} \backslash(0, \ldots, 0) \text {. }
\end{aligned}
$$

1991 Mathematics Subject Classification 32A27, 32A25, 32C30

ANNALES SCIENTIFIQUES DE L'ÉCOLE NORMALE SUPÉRIEURE. - 0012-9593/01/01/@ 2001 Éditions scientifiques et médicales Elsevier SAS. All rights reserved 
Then, it is a classical result that goes back to Jacobi [19], the set $V(P):=\left\{P_{1}=\cdots=P_{n}=0\right\}$ is finite, with cardinal number equal to $\operatorname{deg} P_{1} \cdots \operatorname{deg} P_{n}$ and for any $Q \in \mathbb{C}\left[X_{1}, \ldots, X_{n}\right]$, such that

$$
\operatorname{deg} Q \leqslant \sum_{j=1}^{n} \operatorname{deg}\left(P_{j}\right)-n-1
$$

one has

$$
\operatorname{Res}\left[\begin{array}{c}
Q\left(X_{1}, \ldots, X_{n}\right) \mathrm{d} X \\
P_{1}, \ldots, P_{n}
\end{array}\right]=\sum_{\alpha \in V(P)} \operatorname{Res}_{\alpha}\left[\frac{Q \mathrm{~d} \zeta}{P_{1} \cdots P_{n}}\right]=0 .
$$

Here $\mathrm{d} X$ stands as usual for $\mathrm{d} X_{1} \wedge \cdots \wedge \mathrm{d} X_{n}$ and the residue of the meromorphic form $\frac{Q \mathrm{~d} \zeta}{P_{1} \cdots P_{n}}$ at the isolated point $\alpha \in\left\{P_{1}=\cdots=P_{n}=0\right\}$ is defined as

$$
\operatorname{Res}_{\alpha}\left[\frac{Q \mathrm{~d} \zeta}{P_{1} \cdots P_{n}}\right]=\frac{1}{(2 \pi i)^{n}} \int_{\substack{\left|f_{1}\right|=\varepsilon_{1} \\ \mid f_{n} \cdots=\varepsilon_{n} \\ \zeta \in U_{\alpha}}} \frac{Q(\zeta) \mathrm{d} \zeta}{P_{1}(\zeta) \cdots P_{n}(\zeta)},
$$

where $U_{\alpha}$ is any bounded domain in $\mathbb{C}^{n}$ such that $\{\alpha\}=U_{\alpha} \cap\left\{P_{1}=\cdots=P_{n}=0\right\}$ and the orientation for the cycle $\left\{\zeta \in U_{\alpha}, \| f_{1}\left|=\varepsilon_{1}, \ldots,\right| f_{n} \mid=\varepsilon_{n}\right\}$ is the one that respects the positivity of the differential form $\mathrm{d} \arg \left(f_{1}\right) \wedge \cdots \wedge \mathrm{d} \arg \left(f_{n}\right)$.

The result of Jacobi has a toric pendant which is due to Khovanskii [22]. Let $\mathbb{T}^{n}=\left(\mathbb{C}^{*}\right)^{n}$ and $F_{1}, \ldots, F_{n}$ be $n$ Laurent polynomials in $n$ variables

$$
F_{j}\left(X_{1}, \ldots, X_{n}\right)=\sum_{\alpha_{j} \in \mathcal{A}_{j}} c_{j, \alpha_{j}} X_{1}^{\alpha_{j 1}} \cdots X_{n}^{\alpha_{j n}}, \quad j=1, \ldots, n
$$

with $c_{j, \alpha_{j}} \neq 0$ for any $j \in\{1, \ldots, n\}$, any $\alpha_{j} \in \mathcal{A}_{j}$ (the $\mathcal{A}_{j}$ are the supports of the $F_{j}$ ). Let $\Delta_{j}$ be the Newton polyhedron of $F_{j}$, which is by definition the closed convex hull of $\mathcal{A}_{j}$ in $\mathbb{R}^{n}$. We now impose the Bernstein condition [3], that is:

$$
\begin{aligned}
& \text { for any } \xi \in \mathbb{R}^{n} \backslash(0, \ldots, 0) \text {, the intersection with } \mathbb{T}^{n} \text { of the set } \\
& \left\{\zeta ; \sum_{\substack{\alpha_{j} \in \mathcal{A}_{j} \\
\left\langle\alpha_{j}, \xi\right\rangle=\min _{\eta \in \Delta_{j}}\langle\eta, \xi\rangle}} c_{j, \alpha_{j}} \zeta_{1}^{\alpha_{j 1}} \cdots \zeta_{n}^{\alpha_{j n}}=0, j=1, \ldots, n\right\} \text { is empty. }
\end{aligned}
$$

Under this hypothesis, Bernstein proved in [3] that the set $V^{*}(F):=\left\{F_{1}=\cdots=F_{n}=0\right\} \cap \mathbb{T}^{n}$ is finite with cardinality equal $n$ ! times the Minkowski mixed volume of $\Delta_{1}, \ldots, \Delta_{n}$ and Khovanskii [22] proved that for any Laurent polynomial $Q$ whose support lies in the relative interior of the convex polyhedron $\Delta_{1}+\cdots+\Delta_{n}$, one has

$$
\operatorname{Res}\left[\begin{array}{c}
Q\left(X_{1}, \ldots, X_{n}\right) \mathrm{d} X \\
F_{1}, \ldots, F_{n}
\end{array}\right]_{\mathbb{T}}:=\sum_{\alpha \in V^{*}(F)} \operatorname{Res}_{\alpha}\left[\frac{Q}{F_{1} \cdots F_{n}} \frac{\mathrm{d} \zeta}{\zeta_{1} \cdots \zeta_{n}}\right]=0
$$

(see also [15], Corollary 4.8).

We will see in Section 2 how it is essential to interpret both geometrically and analytically the conditions (1.2) imposed on $\left(P_{1}, \ldots, P_{n}\right)$ in the projective setting or the conditions (1.3) imposed on $\left(F_{1}, \ldots, F_{n}\right)$ in the toric setting. 
In the first case (that is the projective one), the set of conditions (1.2) is geometrically equivalent to the fact that the $n$ Cartier divisors $\mathcal{D}_{1}, \ldots, \mathcal{D}_{n}$, defined on $\mathbb{P}^{n}$ by the homogeneous polynomials ${ }^{\mathrm{h}} P_{j}\left(X_{0}, \ldots, X_{n}\right), j=1, \ldots, n$, are such that their supports $\left|\mathcal{D}_{j}\right|$ satisfy

$$
\left|\mathcal{D}_{1}\right| \cap \cdots \cap\left|\mathcal{D}_{n}\right| \subset \mathbb{C}^{n}
$$

From the analytic point of view, this is equivalent to the following strong properness condition on the polynomial map $P=\left(P_{1}, \ldots, P_{n}\right)$ from $\mathbb{C}^{n}$ to $\mathbb{C}^{n}$ : there are constants $R>0, c>0$, such that, for $\|\zeta\| \geqslant R$,

$$
\sum_{j=1}^{n} \frac{\left|P_{j}(\zeta)\right|}{\left(1+\|\zeta\|^{2}\right)^{\operatorname{deg} P_{j} / 2}} \geqslant c .
$$

In the toric case, given a smooth toric variety $\mathcal{X}$ associated to any fan which is a simple refinement of the fan attached to the polyhedron $\Delta_{1}+\cdots+\Delta_{n}$, conditions (1.3) mean that the effective Cartier divisors

$$
\mathcal{D}_{j}=\operatorname{div}\left(F_{j}\right)+E\left(\Delta_{j}\right)
$$

where $E\left(\Delta_{j}\right)$ is the $\mathbb{T}$-Cartier divisor on $\mathcal{X}$ associated with the polyhedron $\Delta_{j}$ (see [17]), are such that

$$
\left|\mathcal{D}_{1}\right| \cap \cdots \cap\left|\mathcal{D}_{n}\right| \subset \mathbb{T}^{n}
$$

The analytic interpretation of this is the following: there exist constants $R>0, c>0$ such that, for $\zeta \in \mathbb{C}^{n}$ such that $\|\operatorname{Re} \zeta\| \geqslant R$,

$$
\sum_{j=1}^{n} \frac{\left|F_{j}\left(\mathrm{e}^{\zeta_{1}}, \ldots, \mathrm{e}^{\zeta_{n}}\right)\right|}{\mathrm{e}^{H_{\Delta_{j}}(\operatorname{Re} \zeta)}} \geqslant c
$$

where $H_{\Delta_{j}}$ denotes the support function of the convex polyhedron $\Delta_{j}$, that is the function from $\mathbb{R}^{n}$ to $\mathbb{R}$ defined as

$$
H_{\Delta_{j}}(x):=\sup _{\xi \in \Delta_{j}}\langle x, \xi\rangle, \quad x \in \mathbb{R}^{n} .
$$

In [5-7], one used extensively the fact that an analogous version of (1.2) could be stated whenever the polynomial map

$$
P=\left(P_{1}, \ldots, P_{n}\right): \mathbb{C}^{n} \longmapsto \mathbb{C}^{n}
$$

was proper. We will prove in Section 3 of this paper what appears to be the sharpest version of such a result, namely:

THEOREM 1.1. - Let $P=\left(P_{1}, \ldots, P_{n}\right)$ be a polynomial map from $\mathbb{C}^{n}$ to $\mathbb{C}^{n}$ such that there exist constants $c>0, R>0$, and rational numbers $0<\delta_{j} \leqslant \operatorname{deg}\left(P_{j}\right), j=1, \ldots, n$, in order that, for $\|\zeta\| \geqslant R$,

$$
\sum_{j=1}^{n} \frac{\left|P_{j}(\zeta)\right|}{\left(1+\|\zeta\|^{2}\right)^{\delta_{j} / 2}} \geqslant c .
$$

Then, for any polynomial $Q \in \mathbb{C}\left[X_{1}, \ldots, X_{n}\right]$ which satisfies

$$
\operatorname{deg} Q<\delta_{1}+\cdots+\delta_{n}-n,
$$


one has

$$
\operatorname{Res}\left[\begin{array}{c}
Q\left(X_{1}, \ldots, X_{n}\right) \mathrm{d} X \\
P_{1}, \ldots, P_{n}
\end{array}\right]=0 .
$$

We will also prove in the same section the corresponding toric version, namely:

THEOREM 1.2. - Let $F=\left(F_{1}, \ldots, F_{n}\right)$ be a system of Laurent polynomials in $n$ variables, with respective Newton polyhedra $\Delta_{1}, \ldots, \Delta_{n}$. Suppose there exist constants $c>0, R>0$, and convex polyhedra $\delta_{1}, \ldots, \delta_{n}$ with vertices in $\mathbb{Q}^{n}$, with $\delta_{j} \subset \Delta_{j}, j=1, \ldots, n$, and

$$
\operatorname{dim}\left(\delta_{1}+\cdots+\delta_{n}\right)=n,
$$

which are such that, for any $\zeta \in \mathbb{C}^{n}$ with $\|\operatorname{Re} \zeta\| \geqslant R$,

$$
\sum_{j=1}^{n} \frac{\left|F_{j}\left(\mathrm{e}^{\zeta_{1}}, \ldots, \mathrm{e}^{\zeta_{n}}\right)\right|}{\mathrm{e}^{H_{\delta_{j}}(\operatorname{Re} \zeta)}} \geqslant c
$$

Then, for any Laurent polynomial $Q$ such that the support of $Q$ lies in the interior of the convex polyhedron $\delta_{1}+\cdots+\delta_{n}$, one has

$$
\operatorname{Res}\left[\begin{array}{c}
Q\left(X_{1}, \ldots, X_{n}\right) \mathrm{d} X \\
F_{1}, \ldots, F_{n}
\end{array}\right]_{\mathbb{T}}=0
$$

The main tool to be used in the proofs of both theorems will be the Bochner-Martinelli integral formula suitably adapted to each case.

From the point of view of algebraic geometry such theorems are not classical in nature since the supports of the Cartier divisors $\mathcal{D}_{1}, \ldots, \mathcal{D}_{n}$ on $\mathbb{P}^{n}$ corresponding to the ${ }^{\mathrm{h}} P_{j}$ in the first case, or the supports of the divisors $\mathcal{D}_{j}=\operatorname{div}\left(F_{j}\right)+E\left(\Delta_{j}\right)$ on a convenient smooth toric variety $\mathcal{X}$ in the second case, do not intersect properly in $\mathbb{P}^{n}$ or in $\mathcal{X}$ (the intersection is assumed to be proper in $\mathbb{C}^{n}$ or in $\mathbb{T}^{n}$ ). Following the point of view developed by Kollár in [24,25], or by Lazarsfeld and Ein in [13], we will also present in Section 3 a geometric interpretation of the conditions (1.7) (in the projective case) and (1.9) (in the toric case). We will see that the Bochner-Martinelli representation formula we use below fits with the construction of residue currents in the noncomplete intersection case, as proposed in [28]. A better understanding of our two theorems will then rely on the fact that, if $f_{1}, \ldots, f_{n}$ are $n$ holomorphic functions in some domain $\Omega$ of $\mathbb{C}^{n}$, a crucial property of the distribution $T_{f} \in \mathcal{D}^{\prime}(\Omega)$ whose action on a test function $\varphi \in \mathcal{D}(\Omega)$ is defined (see for example [28]) by

$$
T_{f}(\varphi):=\lim _{\varepsilon \rightarrow 0} \frac{1}{\varepsilon^{n}} \int_{\left|f_{1}\right|^{2}+\cdots+\left|f_{n}\right|^{2}=\varepsilon} \sum_{k=1}^{n}(-1)^{k-1} \bar{f}_{k} \bigwedge_{\substack{\ell=1 \\ \ell \neq k}}^{n} \overline{\partial f_{\ell}} \wedge \varphi \mathrm{d} \zeta
$$

is that it is annihilated, as a distribution, by any holomorphic function in $\Omega$ which is locally in the integral closure of the ideal $\left(f_{1}, \ldots, f_{n}\right)^{n}$ (this ideal is contained in $\left(f_{1}, \ldots, f_{n}\right)$ by the classical result of Briançon and Skoda [4]). Therefore, once the hypothesis will be settled in a natural geometric context, our two theorems will appear to be in close relation with this Briançon-Skoda theorem, which also plays a significant role in [25,13], as a transition tool between Lojasiewicz inequalities (or regular separation conditions) and effective versions of the algebraic Nullstellensatz. 
As a consequence, it will then be natural to present in Section 4 some applications of our two theorems to effectivity questions related to the algebraic Nullstellensatz in the projective case or the sparse Nullstellensatz in the toric case, under some properness assumptions on the data in $\mathbb{C}^{n}$ or in $\mathbb{T}^{n}$. Such results will extend or sharpen some previous results in $[6,7,16,31]$. We will also suggest possible applications to some results of Cayley-Bacharach type (see [14]), in the context of improper intersections on $\mathbb{P}^{n}$ or on a smooth toric variety $\mathcal{X}$.

\section{An analytic interpretation of Jacobi or Bernstein conditions}

Using the notation of the previous section we will state in analytic terms the conditions (1.2) or (1.3). We begin with the:

Proposition 2.1. - Let $P_{1}, \ldots, P_{n}$ be $n$ polynomials in $\mathbb{C}\left[X_{1}, X_{2}, \ldots, X_{n}\right]$. The following two assertions are equivalent:

(i) $\left\{\zeta \in \mathbb{C}^{n+1},{ }^{\mathrm{h}} P_{1}=\cdots={ }^{\mathrm{h}} P_{n}=\zeta_{0}=0\right\}=\{0\}$,

(ii) there exist strictly positive constants $R, c$ such that, for any $\zeta \in \mathbb{C}^{n}$ with $\|\zeta\| \geqslant R$,

$$
\sum_{j=1}^{n} \frac{\left|P_{j}(\zeta)\right|}{\left(1+\|\zeta\|^{2}\right)^{\operatorname{deg} P_{j} / 2}} \geqslant c
$$

Proof. - Writing (ii) in homogeneous coordinates, we get that, if $\left(\zeta_{0}, \ldots, \zeta_{n}\right) \in \mathbb{C}^{n+1}$ is such that $\left|\zeta_{1}\right|+\cdots+\left|\zeta_{n}\right|>R\left|\zeta_{0}\right|$, one has

$$
\sum_{j=1}^{n}\left|{ }^{\mathrm{h}} P_{j}\left(\zeta_{0}, \zeta_{1}, \ldots, \zeta_{n}\right)\right| \geqslant c\left(\sum_{j=1}^{n}\left(\left|\zeta_{0}\right|^{2}+\cdots+\left|\zeta_{n}\right|^{2}\right)^{\operatorname{deg} P_{j} / 2}\right)
$$

In particular,

$$
\sum_{j=1}^{n}\left|{ }^{\mathrm{h}} P_{j}\left(0, \zeta_{1}, \ldots, \zeta_{n}\right)\right| \geqslant c\left(\sum_{j=1}^{n}\left(\left|\zeta_{1}\right|^{2}+\cdots+\left|\zeta_{n}\right|^{2}\right)^{\operatorname{deg} P_{j} / 2}\right) .
$$

This shows that (ii) implies (i).

Let now $P_{j}(X)=p_{j}(X)+q_{j}(X)$, such that $\operatorname{deg} q_{j}<\operatorname{deg} p_{j}, p_{j}$ being an homogeneous polynomial with degree $d_{j}=\operatorname{deg}\left(P_{j}\right)$ (the leading terms in $P_{j}$ ). Condition (i) is equivalent to the fact that

$$
\left\{\zeta \in \mathbb{C}^{n}, p_{1}(\zeta)=\cdots=p_{n}(\zeta)=0\right\}=\{(0, \ldots, 0)\} .
$$

Since $p_{1}, \ldots, p_{n}$ are homogeneous with respective degrees $d_{1}, \ldots, d_{n}$, there exists $\widetilde{c}>0$ such that, for any $\zeta \in\left(\mathbb{C}^{n}\right)^{*}$,

$$
\sum_{j=1}^{n} \frac{\left|p_{j}(\zeta)\right|}{\|\zeta\|^{d_{j}}}>\widetilde{c}
$$

Therefore, for any $\zeta \in\left(\mathbb{C}^{n}\right)^{*}$, one has

$$
\sum_{j=1}^{n} \frac{\left|P_{j}(\zeta)\right|}{\|\zeta\|^{d_{j}}} \geqslant \sum_{j=1}^{n} \frac{\left|p_{j}(\zeta)\right|}{\|\zeta\|^{d_{j}}}-\sum_{j=1}^{n} \frac{\left|q_{j}(\zeta)\right|}{\|\zeta\|^{d_{j}}} .
$$


For $\|\zeta\| \geqslant R$, with $R>0$ large enough, one has, since $\operatorname{deg} q_{j}<d_{j}, j=1, \ldots, n$, that

$$
\sum_{j=1}^{n} \frac{\left|q_{j}(\zeta)\right|}{\|\zeta\|^{d_{j}}}<\frac{\widetilde{c}}{2}
$$

Therefore, for $\|\zeta\| \geqslant R$, we have

$$
\sum_{j=1}^{n} \frac{\left|P_{j}(\zeta)\right|}{\|\zeta\|^{d_{j}}} \geqslant \frac{\widetilde{c}}{2} .
$$

The last inequality implies (ii) with some constant $c=c(R)$.

Note that, if $P$ is a polynomial map from $\mathbb{C}^{n}$ to $\mathbb{C}^{n}$, the fact that

$$
\lim _{\|\zeta\| \rightarrow+\infty}\|P(\zeta)\|=+\infty
$$

(which means just that the map is a proper polynomial map in the topological sense) does not imply the strong properness condition (2.1). For example, if $n=2$, the polynomial map $\left(X_{1} X_{2},\left(X_{1}+1\right)\left(X_{2}+1\right)\right)$ is proper, but does not satisfy (2.1) since there are two common zeroes at infinity.

In order to weaken condition (2.1), we introduce the following concept:

Definition 2.1. - Let $\left(P_{1}, \ldots, P_{n}\right)$ be a polynomial map from $\mathbb{C}^{n}$ to $\mathbb{C}^{n}$ and $\left(\delta_{1}, \ldots, \delta_{n}\right)$ be a set of strictly positive rational numbers with $0<\delta_{j} \leqslant \operatorname{deg} P_{j}$ for any $j$. Then we say that $\left(P_{1}, \ldots, P_{n}\right)$ is $\left(\delta_{1}, \ldots, \delta_{n}\right)$-proper if and only if there exist $c>0, R>0$ such that, for any $\zeta \in \mathbb{C}^{n}$ such that $\|\zeta\| \geqslant R$,

$$
\sum_{j=1}^{n} \frac{\left|P_{j}(\zeta)\right|}{\left(1+\|\zeta\|^{2}\right)^{\delta_{j} / 2}} \geqslant c .
$$

Example 2.1. - When $n=2$, the polynomial map $\left(X_{1} X_{2},\left(X_{1}+1\right)\left(X_{2}+1\right)\right)$ is $(1,1)$-proper.

Remark 2.1. - We may extend this notion to the case when the $\delta_{j}$ are rational numbers with the sole conditions $\delta_{j} \leqslant \operatorname{deg} P_{j}$. In this setting, a polynomial map which is $\left(\delta_{1}, \ldots, \delta_{n}\right)$-proper is not necessarily proper in the topological sense.

Let us now formulate the toric analogue of the Proposition 2.1.

PROPOSITION 2.2.-Let $F_{1}, \ldots, F_{n}$ be $n$ Laurent polynomials with Newton polyhedra $\Delta_{1}, \ldots, \Delta_{n}$. The following two assertions are equivalent:

(i) $F_{1}, \ldots, F_{n}$ satisfy the Bernstein conditions (1.3);

(ii) there exist strictly positive constants $R$, c such that, for any $\zeta \in \mathbb{C}^{n}$, with $\|\operatorname{Re} \zeta\| \geqslant R$,

$$
\sum_{j=1}^{n} \frac{\left|F_{j}\left(\mathrm{e}^{\zeta_{1}}, \ldots, \mathrm{e}^{\zeta_{n}}\right)\right|}{\mathrm{e}^{H_{\Delta_{j}}(\operatorname{Re} \zeta)}} \geqslant c
$$

Proof. - We first prove that (i) implies (ii). Let us assume that $\left(F_{1}, \ldots, F_{n}\right)$ satisfy the Bernstein conditions (1.3). In order to prove (ii), it is enough to show that one can find a conic open sector $S_{u}$ in $\mathbb{R}^{n}$ containing $-u$ and strictly positive constants $R_{u}, c_{u}$, such that, for any 
$\zeta \in \mathbb{C}^{n}$ with $\operatorname{Re} \zeta \in S_{u}$ and $\|\operatorname{Re} \zeta\| \geqslant R_{u}$, one has

$$
\sum_{j=1}^{m} \frac{\left|F_{j}\left(\mathrm{e}^{\zeta_{1}}, \ldots, \mathrm{e}^{\zeta_{n}}\right)\right|}{\mathrm{e}^{H_{\Delta_{j}}(\operatorname{Re} \zeta)}} \geqslant c_{u} .
$$

Then, if one can do so for each rational $u$, the existence of positive constants $R$ and $c$ will follow from a compactness argument.

Applying in the $\zeta$-space a change of coordinates $\zeta^{\prime}=A \zeta, A \in \mathrm{GL}_{n}(\mathbb{Z})$, we may assume that $u=(1,0, \ldots, 0)=e_{1}$. Let us write, for $j=1, \ldots, n$,

$$
F_{j}\left(\mathrm{e}^{\zeta_{1}}, \ldots, \mathrm{e}^{\zeta_{n}}\right)=\mathrm{e}^{k_{j} \zeta_{1}} f_{j}\left(\mathrm{e}^{\zeta_{2}}, \ldots, \mathrm{e}^{\zeta_{n}}\right)+\widetilde{F}_{j}\left(\mathrm{e}^{\zeta_{1}}, \ldots, \mathrm{e}^{\zeta_{n}}\right),
$$

where the support of $\widetilde{F}_{j}$ is included in $\left\{x_{1}>k_{j}\right\}$. As noticed by Kazarnovskii [20] (see also [27], Section 2, from which we got our inspiration here), the fact that Bernstein conditions (1.3) are satisfied for $\left(F_{1}, \ldots, F_{n}\right)$ is equivalent to the following fact: for any set of respective faces $\left(\gamma_{1}, \ldots, \gamma_{n}\right)$ of the Newton polyhedra $\Delta_{1}, \ldots, \Delta_{n}$ of $F_{1}, \ldots, F_{n}$, there exists $\varepsilon\left(\gamma_{1}, \ldots, \gamma_{n}\right)>0$ such that, for any $\left(\zeta_{1}, \ldots, \zeta_{n}\right) \in \mathbb{C}^{n}$,

$$
\sum_{j=1}^{n} \frac{\left|F_{j}^{\gamma_{j}}\left(\mathrm{e}^{\zeta_{1}}, \ldots, \mathrm{e}^{\zeta_{n}}\right)\right|}{\mathrm{e}^{H_{\gamma_{j}}\left(\operatorname{Re} \zeta_{1}, \ldots, \operatorname{Re} \zeta_{n}\right)}} \geqslant \varepsilon\left(\gamma_{1}, \ldots, \gamma_{n}\right),
$$

where, for each $j=1, \ldots, n, F_{j}^{\gamma_{j}}$ denotes the part obtained from $F_{j}$ by keeping only monomials corresponding to points in $\gamma_{j}$ and deleting all the others. It is clear that whenever $\delta_{j}$ denotes the Newton polyhedron of $f_{j}$ (considered as a Laurent polynomial in $n-1$ variables with support in the subspace $e_{1}^{\perp}$ of $\mathbb{R}^{n}$ ), the convex sets $\widetilde{\delta}_{j}=\delta_{j}+k_{j} e_{1}, j=1, \ldots, n$, are respective faces of $\Delta_{1}, \ldots, \Delta_{n}$. Therefore, one has, for some $\varepsilon>0$, for $\left(\zeta_{1}, \ldots, \zeta_{n}\right) \in \mathbb{C}^{n}$,

$$
\sum_{j=1}^{n} \frac{\left|\mathrm{e}^{k_{j} \zeta_{1}} f_{j}\left(\mathrm{e}^{\zeta_{2}}, \ldots, \mathrm{e}^{\zeta_{n}}\right)\right|}{\mathrm{e}^{H_{\widetilde{\delta}_{j}}\left(\operatorname{Re} \zeta_{1}, \ldots, \operatorname{Re} \zeta_{n}\right)}} \geqslant \varepsilon .
$$

Since the support of $\widetilde{F}_{j}$ in (2.5) is included in $\left\{x_{1}>k_{j}\right\}$, there exists $\rho>0$, such that, for any $\zeta=\left(\zeta_{1}, \ldots, \zeta_{n}\right)$ with $\operatorname{Re} \zeta_{1}<0$ and $\left|\operatorname{Re} \zeta_{j}\right| \leqslant \rho\left|\operatorname{Re} \zeta_{1}\right|$ for $j=2, \ldots, n$, one has

$$
H_{\widetilde{\delta}_{j}}(\operatorname{Re} \zeta)=H_{\Delta_{j}}(\operatorname{Re} \zeta), \quad j=1, \ldots, n .
$$

On the other hand, if $\rho$ is small enough, then there exists $R>0$ such that for any $\zeta \in \mathbb{C}^{n}$ with $\operatorname{Re} \zeta_{1} \leqslant-R$ and $\left|\operatorname{Re} \zeta_{j}\right| \leqslant \rho\left|\operatorname{Re} \zeta_{1}\right|$ for $j=2, \ldots, n$, one has

$$
\sum_{j=1}^{n} \frac{\left|\widetilde{F}_{j}\left(\mathrm{e}^{\zeta_{1}}, \ldots, \mathrm{e}^{\zeta_{n}}\right)\right|}{\mathrm{e}^{H_{\tilde{\delta}_{j}}\left(\operatorname{Re} \zeta_{1}, \ldots, \operatorname{Re} \zeta_{n}\right)}}<\frac{\varepsilon}{2} .
$$

From (2.6), (2.7) and (2.8), we get that for $\zeta$ in the conic sector

$$
S_{u}:=\left\{\operatorname{Re} \zeta_{1}<0,\left|\operatorname{Re} \zeta_{j}\right|<\rho\left|\operatorname{Re} \zeta_{1}\right|, j=2, \ldots, n\right\},
$$

the inequality (2.4) is valid for $\|\operatorname{Re} \zeta\| \geqslant R=R_{u}$ and $c_{u}=\varepsilon / 2$. This shows that (ii) holds for the system $\left(F_{1}, \ldots, F_{n}\right)$. 
In order to prove the converse direction we will construct a globally defined real analytic function that is not vanishing in $\mathcal{X} \backslash \mathbb{T}$. This is done as follows:

For each $j \in\{1, \ldots, n\}$ choose $n$ Laurent polynomials $\left(G_{1}^{(j)}, \ldots, G_{n}^{(j)}\right)$ with Newton polyhedron $\Delta_{j}$ such that the system $\left(G_{1}^{(j)}, \ldots, G_{n}^{(j)}\right)$ satisfies the Bernstein conditions (1.3). It follows from the fact that (i) implies (ii) that, for some convenient constants $C_{j} \geqslant c_{j}>0$, $R_{j}>0$, one has, for any $\zeta \in \mathbb{C}^{n}$ with $\|\operatorname{Re} \zeta\| \geqslant R_{j}$,

$$
c_{j} \mathrm{e}^{H_{\Delta_{j}}(\operatorname{Re} \zeta)} \leqslant \sum_{k=1}^{n}\left|G_{k}^{(j)}\left(\mathrm{e}^{\zeta_{1}}, \ldots, \mathrm{e}^{\zeta_{n}}\right)\right| \leqslant C_{j} \mathrm{e}^{H_{\Delta_{j}}(\operatorname{Re} \zeta)} .
$$

Consider now on the torus $\mathbb{T}^{n}$ the real analytic function

$$
\zeta \longmapsto \phi(\zeta):=\sum_{j=1}^{n} \frac{\left|F_{j}(\zeta)\right|^{2}}{\sum_{k=1}^{n}\left|G_{k}^{(j)}(\zeta)\right|^{2}} .
$$

Let $\mathcal{X}$ be any toric variety associated to a simple refinement of the fan which corresponds to $\Delta_{1}+\cdots+\Delta_{n}$. The Laurent polynomials $\left(G_{1}^{(j)}, \ldots, G_{n}^{(j)}\right)$ induce effective Cartier divisors $\left(\mathcal{D}_{1}^{(j)}, \ldots, \mathcal{D}_{n}^{(j)}\right)$ on $\mathcal{X}$, namely

$$
\mathcal{D}_{k}^{(j)}=\operatorname{div}\left(G_{k}^{(j)}\right)+E\left(\Delta_{j}\right), \quad 1 \leqslant j, k \leqslant n,
$$

where $E\left(\Delta_{j}\right)$ is the $\mathbb{T}$-Cartier divisor on $\mathcal{X}$ corresponding to $\Delta_{j}$ (it is well defined, since $\mathcal{X}$ corresponds to a fan which is compatible with $\left.\Delta_{j}\right)$. The fact that the system $\left(G_{1}^{(j)}, \ldots, G_{n}^{(j)}\right)$ obeys the Bernstein conditions is equivalent (see for example [17]) to

$$
\left|\mathcal{D}_{1}^{(j)}\right| \cap \cdots \cap\left|\mathcal{D}_{n}^{(j)}\right|=\mathcal{L}_{j} \subset \mathbb{T}^{n}
$$

For homogeneity reasons, the function

$$
\zeta \longmapsto \phi\left(\zeta_{1}, \ldots, \zeta_{n}\right)
$$

extends from $\mathbb{T}^{n} \backslash \bigcup_{j=1}^{n} \mathcal{L}_{j}$ to a function $\widetilde{\phi}$ defined globally as a real analytic function on $\mathcal{X} \backslash \bigcup_{j=1}^{n} \mathcal{L}_{j}$

Now we are ready to complete the proof of the final step. Assume that $\left(F_{1}, \ldots, F_{n}\right)$ satisfies (ii). For

$$
\left|\zeta_{1}\right|+\cdots+\left|\zeta_{n}\right|+\frac{1}{\left|\zeta_{1}\right|}+\cdots+\frac{1}{\left|\zeta_{n}\right|}
$$

large enough, we have, for some constants $0<\widetilde{c}<\widetilde{C}<\infty$,

$$
\widetilde{c} \leqslant\left|\widetilde{\phi}\left(\zeta_{1}, \ldots, \zeta_{n}\right)\right|=\left|\phi\left(\zeta_{1}, \ldots, \zeta_{n}\right)\right| \leqslant \widetilde{C} .
$$

Therefore $\widetilde{\phi}$ does not vanish on $\mathcal{X} \backslash \mathbb{T}^{n}$, which implies that the effective Cartier divisors $\mathcal{D}_{j}$ induced by the $F_{j}$ on $\mathcal{X}$ by

$$
\mathcal{D}_{j}=\operatorname{div}\left(F_{j}\right)+E\left(\Delta_{j}\right)
$$


are such that

$$
\left|\mathcal{D}_{1}\right| \cap \cdots \cap\left|\mathcal{D}_{n}\right| \subset \mathbb{T}^{n}
$$

This is equivalent to say that the Bernstein conditions are fulfilled for the system $\left(F_{1}, \ldots, F_{n}\right)$.

In order to weaken the properness condition (2.2), we introduce the toric analogue of Definition 2.1.

Definition 2.2. - Let $\left(F_{1}, \ldots, F_{n}\right)$ be a system of Laurent polynomials in $n$ variables, with Newton polyhedra $\Delta_{1}, \ldots, \Delta_{n}$, and $\left(\delta_{1}, \ldots, \delta_{n}\right)$ be a collection of closed convex polyhedra with vertices in $\mathbb{Q}^{n}$, with $\delta_{j} \subset \Delta_{j}, j=1, \ldots, n$. Then, we say that $\left(F_{1}, \ldots, F_{n}\right)$ is $\left(\delta_{1}, \ldots, \delta_{n}\right)$-proper if and only if there exist $c>0, R>0$ such that, for any $\zeta \in \mathbb{C}^{n}$ such that $\|\operatorname{Re} \zeta\| \geqslant R$,

$$
\sum_{j=1}^{n} \frac{\left|F_{j}\left(\mathrm{e}^{\zeta_{1}}, \ldots, \mathrm{e}^{\zeta_{n}}\right)\right|}{\mathrm{e}^{H_{\delta_{j}}(\operatorname{Re} \zeta)}} \geqslant c
$$

Example 2.2. - Let $n=2$ and

$$
\begin{aligned}
& F_{1}=X_{1}^{2} X_{2}^{2}+X_{1}^{2} X_{2}^{-2}+\alpha_{1} X_{1} X_{2}+\beta_{1} X_{1} X_{2}^{-1}+\gamma_{1} X_{2}^{2} X_{1}^{-2}+\delta_{1} X_{1}^{-2} X_{2}^{-2}, \\
& F_{2}=X_{1}^{2} X_{2}^{2}+X_{1}^{2} X_{2}^{-2}+\alpha_{2} X_{1} X_{2}+\beta_{2} X_{1} X_{2}^{-1}+\gamma_{2} X_{2}^{2} X_{1}^{-2}+\delta_{2} X_{1}^{-2} X_{2}^{-2},
\end{aligned}
$$

with the conditions

$$
\begin{gathered}
\gamma_{1}\left(\delta_{1}-\delta_{2}\right)-\delta_{1}\left(\gamma_{1}-\gamma_{2}\right) \neq 0, \\
\left(\alpha_{1}-\alpha_{2}\right)^{2}-\left(\beta_{1}-\beta_{2}\right)^{2} \neq 0 .
\end{gathered}
$$

Then $\left(F_{1}, F_{2}\right)$ is $(\delta, \delta)$-proper, where

$$
\delta=\overline{\operatorname{conv}\{(-2,-2),(2,2),(1,1),(1,-1)\}}
$$

In fact, it is enough to notice that $\left(F_{1}-F_{2}, F_{1}\right)$ satisfy the Bernstein conditions and have as respective Newton polyhedra $\delta$ and $[-2,2] \times[-2,2]$, so that by Proposition 2.2, one has, for $\left\|\left(\operatorname{Re} \zeta_{1}, \operatorname{Re} \zeta_{2}\right)\right\| \geqslant R>0$,

$$
\frac{\left|\left(F_{1}-F_{2}\right)\left(\mathrm{e}^{\zeta_{1}}, \mathrm{e}^{\zeta_{2}}\right)\right|}{\mathrm{e}^{H_{\delta}\left(\operatorname{Re} \zeta_{1}, \operatorname{Re} \zeta_{2}\right)}}+\frac{\left|F_{1}\left(\mathrm{e}^{\zeta_{1}}, \mathrm{e}^{\zeta_{2}}\right)\right|}{\mathrm{e}^{H_{[-2,2]^{2}}\left(\operatorname{Re} \zeta_{1}, \operatorname{Re} \zeta_{2}\right)}} \geqslant c,
$$

which implies, for such $\zeta$,

$$
\frac{\left|F_{1}\left(\mathrm{e}^{\zeta_{1}}, \mathrm{e}^{\zeta_{2}}\right)\right|}{\mathrm{e}^{H_{\delta}\left(\operatorname{Re} \zeta_{1}, \operatorname{Re} \zeta_{2}\right)}}+\frac{\left|F_{2}\left(\mathrm{e}^{\zeta_{1}}, \mathrm{e}^{\zeta_{2}}\right)\right|}{\mathrm{e}^{H_{\delta}\left(\operatorname{Re} \zeta_{1}, \operatorname{Re} \zeta_{2}\right)}} \geqslant \frac{c}{2} .
$$

\section{Proof of the vanishing theorems}

\subsection{The case of the projective space $\mathbb{P}^{n}$}

Our basic tool will be multidimensional residue theory through an approach based on the use of Bochner-Martinelli integral representation formulas. Let us recall here some well known facts.

Let $P_{1}, \ldots, P_{n}$ be $n$ polynomials in $n$ variables defining a discrete (hence finite) variety in $\mathbb{C}^{n}$. It is shown in [28] that if $\alpha \in\left\{P_{1}=\cdots=P_{n}=0\right\}$ and $\varphi \in \mathcal{D}\left(\mathbb{C}^{n}\right)$ is such that $\varphi \equiv 1$ in 
a neighborhood of $\alpha$ and $\varphi \equiv 0$ in a neighborhood of any point in $\left\{P_{1}=\cdots=P_{n}=0\right\} \backslash\{\alpha\}$, then the local residue at the point $\alpha$ is

$$
\begin{aligned}
\operatorname{Res}_{\alpha}\left[\frac{Q \mathrm{~d} \zeta}{P_{1} \cdots P_{n}}\right] & =\gamma_{n} \lim _{\varepsilon \rightarrow 0} \frac{1}{\varepsilon^{n}} \int_{\|P\|^{2}=\varepsilon} Q\left(\sum_{k=1}^{n}(-1)^{k-1} \overline{P_{k}} \bigwedge_{\substack{\ell=1 \\
\ell \neq k}}^{n} \overline{\partial P_{\ell}}\right) \wedge \varphi \mathrm{d} \zeta \\
& =\gamma_{n} \lim _{\varepsilon \rightarrow 0} \int_{\|P\|^{2}=\varepsilon} \frac{Q\left(\sum_{k=1}^{n}(-1)^{k-1} \overline{P_{k}} \bigwedge_{\substack{n=1 \\
\ell \neq k}}^{n} \overline{\partial P_{\ell}}\right) \wedge \varphi \mathrm{d} \zeta}{\|P\|^{2 n}}
\end{aligned}
$$

where as usual $\|P\|^{2}=\left|P_{1}\right|^{2}+\cdots+\left|P_{n}\right|^{2}$ and $\gamma_{n}=\frac{(-1)^{\frac{n(n-1)}{2}}(n-1) !}{(2 \pi i)^{n}}$. Using Stokes's theorem and observing that the form

$$
\frac{Q\left(\sum_{k=1}^{n}(-1)^{k-1} \overline{P_{k}} \bigwedge_{\substack{\ell=1 \\ \ell \neq k}}^{n} \overline{\partial P_{\ell}}\right) \wedge \varphi \mathrm{d} \zeta}{\|P\|^{2 n}}
$$

is closed in a punctured neighborhood $U_{\alpha} \backslash\{\alpha\}$, we get from (3.2) that if $U_{\alpha}$ is small enough and with piecewise smooth boundary,

$$
\operatorname{Res}_{\alpha}\left[\frac{Q \mathrm{~d} \zeta}{P_{1} \cdots P_{n}}\right]=\gamma_{n} \int_{\partial U_{\alpha}} \frac{Q\left(\sum_{k=1}^{n}(-1)^{k-1} \overline{P_{k}} \bigwedge_{\substack{\ell=1 \\ \ell \neq k}}^{n} \overline{\partial P_{\ell}}\right) \wedge \mathrm{d} \zeta}{\|P\|^{2 n}} .
$$

Therefore, if $U$ is any bounded open set with smooth boundary containing in its interior the set $V(P):=\left\{P_{1}=\cdots=P_{n}=0\right\}$, then the global residue is the sum of the local residues, that is

$$
\operatorname{Res}\left[\begin{array}{c}
Q\left(X_{1}, \ldots, X_{n}\right) \mathrm{d} X \\
P_{1}, \ldots, P_{n}
\end{array}\right]=\gamma_{n} \int_{\partial U} \frac{Q\left(\sum_{k=1}^{n}(-1)^{k-1} \overline{P_{k}} \bigwedge_{\substack{\ell=1 \\
\ell \neq k}}^{n} \overline{\partial P_{\ell}}\right) \wedge \mathrm{d} \zeta}{\|P\|^{2 n}} .
$$

We can rewrite (3.3) as follows: if

$$
s_{0}=\left(\frac{\overline{P_{1}}}{\|P\|^{2}}, \ldots, \frac{\overline{P_{n}}}{\|P\|^{2}}\right)=\left(s_{01}, \ldots, s_{0 n}\right),
$$

then

$$
\operatorname{Res}\left[\begin{array}{c}
Q\left(X_{1}, \ldots, X_{n}\right) \mathrm{d} X \\
P_{1}, \ldots, P_{n}
\end{array}\right]=\gamma_{n} \int_{\partial U} Q(\zeta)\left(\sum_{k=1}^{n}(-1)^{k-1} s_{0 k} \mathrm{~d} s_{0,[k]}\right) \wedge \mathrm{d} \zeta,
$$

where $\mathrm{d} s_{0,[k]}:=\bigwedge_{j \neq k} \mathrm{~d} s_{0 j}, k=1, \ldots, n$.

An homotopy argument shows that one can replace the vector-function $s_{0}$ above by any vectorfunction $s$, which is $\mathrm{C}^{1}$ in a neighborhood of the $\partial U$ and satisfies

$$
\langle s(\zeta), P(\zeta)\rangle=\sum_{k=1}^{n} s_{k}(\zeta) P_{k}(\zeta) \equiv 1, \quad \zeta \in \partial U
$$


Then the global residue is given by the generalized Bochner-Martinelli formula

$$
\operatorname{Res}\left[\begin{array}{c}
Q\left(X_{1} \ldots, X_{n}\right) \mathrm{d} X \\
P_{1}, \ldots, P_{n}
\end{array}\right]=\gamma_{n} \int_{\partial U} Q\left(\sum_{k=1}^{n}(-1)^{k-1} s_{k} \mathrm{~d} s_{[k]}\right) \wedge \mathrm{d} \zeta .
$$

Before proceeding any further we point out that it is enough to prove Theorem 1.1 for the case when $\delta_{j}, j=1, \ldots, n$, are strictly positive integers. In order to do so, it is enough to use the compatibility of the residue calculus with the change of basis (see for example [26], Section 2, Proposition 2.3), which asserts that, for any $N \in \mathbb{N}^{*}$,

$$
\operatorname{Res}\left[\begin{array}{c}
Q(X) \mathrm{d} X \\
P_{1}(X), \ldots, P_{n}(X)
\end{array}\right]=\operatorname{Res}\left[\begin{array}{c}
Q\left(X_{1}^{N}, \ldots, X_{n}^{N}\right)\left(X_{1} \cdots X_{n}\right)^{N-1} \mathrm{~d} X \\
P_{1}\left(X_{1}^{N}, \ldots, X_{n}^{N}\right), \ldots, P_{n}\left(X_{1}^{N}, \ldots, X_{n}^{N}\right)
\end{array}\right]
$$

Let $N$ be a common denominator for the rational numbers $\delta_{j}, j=1, \ldots, n$; then the polynomials

$$
\widetilde{P}_{j}(X)=P_{j}\left(X_{1}^{N}, \ldots, X_{n}^{N}\right), \quad j=1, \ldots, n,
$$

have respective degrees $N \operatorname{deg} P_{j}, j=1, \ldots, n$, and satisfy (2.2) with $\widetilde{\delta}_{j}=N \delta_{j} \in \mathbb{N}^{*}$. If we assume that our result holds when the $\delta_{j}$ are integers, we get that the residue symbol (3.5) is zero when

$$
N \operatorname{deg} Q+n(N-1) \leqslant N\left(\delta_{1}+\cdots+\delta_{n}\right)-n-1,
$$

that is

$$
\operatorname{deg} Q \leqslant \delta_{1}+\cdots+\delta_{n}-n-\frac{1}{N}
$$

Therefore, we have (1.8) whenever

$$
\operatorname{deg} Q<\delta_{1}+\cdots+\delta_{n}-1
$$

as we want. We will assume from now on that $\delta_{j} \in \mathbb{N}^{*}$ for any $j \in\{1, \ldots, n\}$.

The next lemma introduces the Bochner-Martinelli residual currents into the computation of the global residue.

Lemma 3.1. - Let $P=\left(P_{1}, \ldots, P_{n}\right)$ be a polynomial map from $\mathbb{C}^{n}$ to $\mathbb{C}^{n}$ defining a discrete (hence finite) zero variety $V(P)$ in $\mathbb{C}^{n}$. Assume also that $R>0$ is sufficiently large so that $V(P)=\left\{P_{1}=\cdots=P_{n}=0\right\} \subset \mathrm{B}(0, R)$. For $D_{j}:=\operatorname{deg} P_{j}$ pick an integer $M$ large enough, so that

$$
\delta_{k}+M-D_{k}>0, \quad \forall k \in\{1, \ldots, n\} .
$$

Then, if $s_{0}^{\delta, M}$ is the vector function defined on $\mathbb{C}^{n}$ as

$$
s_{0}^{\delta, M}(\zeta):=\left(\frac{\overline{P_{1}(\zeta)}}{\left(1+\|\zeta\|^{2}\right)^{\delta_{1}+M}}, \ldots, \frac{\overline{P_{n}(\zeta)}}{\left(1+\|\zeta\|^{2}\right)^{\delta_{n}+M}}\right)
$$

and

$$
\|P(\zeta)\|_{\delta, M}^{2}:=\sum_{j=1}^{n} \frac{\left|P_{j}(\zeta)\right|^{2}}{\left(1+\|\zeta\|^{2}\right)^{M+\delta_{j}}}
$$


one has, for any $Q \in \mathbb{C}\left[X_{1}, \ldots, X_{n}\right]$,

$$
\operatorname{Res}\left[\begin{array}{c}
Q(X) \mathrm{d} X \\
P_{1}, \ldots, P_{n}
\end{array}\right]=\gamma_{n}\left[\int_{\|\zeta\|=R}\|P\|_{\delta, M}^{2(\lambda-n)} Q\left(\sum_{k=1}^{n}(-1)^{k-1} s_{0 k}^{\delta, M} \mathrm{~d} s_{0,[k]}^{\delta, M}\right) \wedge \mathrm{d} \zeta\right]_{\lambda=0}
$$

Proof. - Let us define the vector function $s=s^{\delta, M}$ in $\mathbb{C}^{n} \backslash V(P)$ as follows

$$
s^{\delta, M}(\zeta)=\frac{1}{\|P(\zeta)\|_{\delta, M}^{2}}\left(\frac{\overline{P_{1}(\zeta)}}{\left(1+\|\zeta\|^{2}\right)^{\delta_{1}+M}}, \ldots, \frac{\overline{P_{n}(\zeta)}}{\left(1+\|\zeta\|^{2}\right)^{\delta_{n}+M}}\right) .
$$

Formula (3.4) implies that

$$
\begin{aligned}
\operatorname{Res}\left[\begin{array}{c}
Q(X) \mathrm{d} X \\
P_{1}, \ldots, P_{n}
\end{array}\right] & =\gamma_{n} \int_{\|\zeta\|=R} Q\left(\sum_{k=1}^{n}(-1)^{k-1} s_{k}^{\delta, M} \mathrm{~d} s_{[k]}^{\delta, M}\right) \wedge \mathrm{d} \zeta \\
& =\gamma_{n} \int_{\|\zeta\|=R}\|P\|_{\delta, M}^{-2 n} Q\left(\sum_{k=1}^{n}(-1)^{k-1} s_{0 k}^{\delta, M} \mathrm{~d} s_{0,[k]}^{\delta, M}\right) \wedge \mathrm{d} \zeta \\
& =\gamma_{n}\left[\int_{\|\zeta\|=R}\|P\|_{\delta, M}^{2(\lambda-n)} Q\left(\sum_{k=1}^{n}(-1)^{k-1} s_{0 k}^{\delta, M} \mathrm{~d} s_{0,[k]}^{\delta, M}\right) \wedge \mathrm{d} \zeta\right]_{\lambda=0} .
\end{aligned}
$$

The last equality completes the proof of the lemma.

Next, we introduce the common zeroes at infinity of the polynomial map $\left(P_{1}, \ldots, P_{n}\right)$ into the description of the action of the Bochner-Martinelli residual current as it is given by (3.6).

For $\lambda$ fixed with $\operatorname{Re} \lambda \gg 1$, let us express in homogeneous coordinates $\widetilde{\zeta}:=\left(\zeta_{0}, \ldots, \zeta_{n}\right)$ the differential form

$$
\|P\|_{\delta, M}^{2(\lambda-n)} Q\left(\sum_{k=1}^{n}(-1)^{k-1} s_{0 k}^{\delta, M} \mathrm{~d} s_{0,[k]}^{\delta, M}\right) \wedge \mathrm{d} \zeta
$$

This leads to a differential $(n, n-1)$-form in $\mathbb{P}^{n}$ (depending on the complex parameter $\lambda$ ), which will be denoted as $\Theta_{P, Q, \lambda}^{\delta, M}$. This form is

$$
\begin{aligned}
\Theta_{P, Q, \lambda}^{\delta, M}(\widetilde{\zeta})= & \left(\frac{\|\mathcal{P}\|_{\delta, M}}{\|\widetilde{\zeta}\|^{|\delta|+M}}\right)^{2(\lambda-n)} \zeta_{0}^{n M+|\delta|-n-1} \mathcal{Q}(\widetilde{\zeta}) \\
& \times\left(\sum_{k=1}^{n}(-1)^{k-1} \frac{\bar{\zeta}^{\delta_{k}+M-D_{k}} \overline{\mathcal{P}_{k}}}{\|\widetilde{\zeta}\|^{2\left(\delta_{[k]}+M\right)}} \bigwedge_{\substack{\ell=1 \\
\ell \neq k}}^{k} \bar{\partial}\left[\frac{\bar{\zeta}_{0}^{\delta_{\ell}+M-D_{\ell}} \overline{\mathcal{P}_{\ell}}}{\|\widetilde{\zeta}\|^{2\left(\delta_{[\ell]}+M\right)}}\right]\right) \wedge \Omega,
\end{aligned}
$$

where $|\delta|=\delta_{1}+\cdots+\delta_{n}, \delta_{[j]}=|\delta|-\delta_{j}$ for $j=1, \ldots, n, \Omega$ is the Euler form, and

$$
\|\mathcal{P}(\widetilde{\zeta})\|_{\delta, M}^{2}:=\sum_{j=1}^{n}\left|\mathcal{P}_{j}\right|^{2}\left|\zeta_{0}\right|^{2\left(\delta_{j}-D_{j}+M\right)}\|\widetilde{\zeta}\|^{2 \delta_{[j]}}
$$

$\mathcal{P}_{1}, \ldots, \mathcal{P}_{n}, \mathcal{Q}$, being the respective homogenizations of $P_{1}, \ldots, P_{n}, Q$; the norm $\|\widetilde{\zeta}\|$ is the Euclidean norm in $\mathbb{C}^{n+1}$. 
Since

$$
\bar{\partial}\left[\|P\|_{\delta, M}^{2(\lambda-n)} Q\left(\sum_{k=1}^{n}(-1)^{k-1} s_{0 k}^{\delta, M} \mathrm{~d} s_{0,[k]}^{\delta, M}\right) \wedge \mathrm{d} \zeta\right]=n \lambda\|P\|_{\delta, M}^{2(\lambda-n)} Q\left(\bigwedge_{k=1}^{n} \bar{\partial} s_{0 k}^{\delta, M}\right) \wedge \mathrm{d} \zeta
$$

we have, if the action of the $\bar{\partial}$ operator is now considered on the projective differential forms (expressed in homogeneous coordinates),

$$
\bar{\partial} \Theta_{P, Q, \lambda}^{\delta, M}=n \lambda\left(\frac{\|\mathcal{P}(\widetilde{\zeta})\|_{\delta, M}}{\|\widetilde{\zeta}\|^{|\delta|+M}}\right)^{2(\lambda-n)} \zeta_{0}^{-\operatorname{deg} Q-n-1} \mathcal{Q}(\widetilde{\zeta}) A_{P, Q}^{\delta, M}\left(\frac{\zeta_{1}}{\zeta_{0}}, \ldots, \frac{\zeta_{n}}{\zeta_{0}}\right) \wedge \Omega(\widetilde{\zeta}),
$$

where

$$
A_{P, Q}^{\delta, M}:=\bigwedge_{k=1}^{n} \bar{\partial} s_{0 k}^{\delta, M}
$$

Since

$$
s_{0 k}^{\delta, M}\left(\frac{\zeta_{1}}{\zeta_{0}}, \ldots, \frac{\zeta_{n}}{\zeta_{0}}\right)=\left|\zeta_{0}\right|^{2\left(\delta_{k}+M\right)} \frac{\overline{\mathcal{P}_{k}(\widetilde{\zeta})} \overline{\zeta_{0}}-D_{k}}{\|\widetilde{\zeta}\|^{2\left(\delta_{k}+M\right)}}
$$

one has

$$
A_{P, Q}^{\delta, M}\left(\zeta_{1} \zeta_{0}, \ldots, \frac{\zeta_{n}}{\zeta_{0}}\right)=\zeta_{0}^{n M+|\delta|} \bigwedge_{k=1}^{n} \bar{\partial}\left[\frac{\bar{\zeta}_{0}^{\delta_{k}+M-D_{k}} \overline{\mathcal{P}_{k}}}{\|\widetilde{\zeta}\|^{2\left(M+\delta_{k}\right)}}\right]
$$

This shows (as a consequence of Atiyah's Theorem [1]) that the map

$$
\lambda \longmapsto \Theta_{P, Q, \lambda}^{\delta, M}
$$

can be considered as a meromorphic map with values in the space of $(n, n-1)$-currents in $\mathbb{P}^{n}(\mathbb{C})$.

At this stage we are ready for the

Proof of the Theorem 1.1. - Take $R>0$ as in the Lemma 3.1 and consider the complement in $\mathbb{P}^{n}(\mathbb{C})$ of $\mathrm{B}(0, R)$ as a $2 n$-chain $\Sigma$ in $\mathbb{P}^{n}$ (with smooth boundary). One has, for $\operatorname{Re} \lambda \gg 1$, using Stokes's theorem

$$
\int_{\partial \Sigma} \Theta_{P, Q, \lambda}^{\delta, M}=\int_{\Sigma} \bar{\partial}\left[\Theta_{P, Q, \lambda}^{\delta, M}\right]
$$

Therefore, one can rewrite (3.6) as

$$
\operatorname{Res}\left[\begin{array}{c}
Q(X) \mathrm{d} X \\
P_{1}, \ldots, P_{n}
\end{array}\right]=-\gamma_{n}\left[\int_{\partial \Sigma} \Theta_{P, Q, \lambda}^{\delta, M}\right]_{\lambda=0}=-\gamma_{n}\left[\int_{\Sigma} \bar{\partial}\left(\Theta_{P, Q, \lambda}^{\delta, M}\right)\right]_{\lambda=0}
$$

(the total sum of residues in $\mathbb{C}^{n}$ equals the opposite of the "residue" at infinity). This identity essentially reduces the proof of the Theorem 1.1 to the computation of an integral describing the "residue" at infinity.

In order to carry out the computation of this integral (and to prove that it vanishes in the situation we are dealing with), we first express the integrant it involves in homogeneous 
coordinates; then we localize the problem and look at the analytic continuation up to the origin of the meromorphic function

$$
\lambda \longmapsto \int_{\Sigma} \varphi \bar{\partial}\left(\Theta_{P, Q, \lambda}^{\delta, M}\right),
$$

when $\varphi$ is an element in $\mathcal{D}\left(\mathbb{P}^{n}(\mathbb{C})\right)$ with support contained in a neighborhood $V$ of some point $x$ at infinity in $\mathbb{P}^{n}(\mathbb{C})$ (these are the only interesting points, since if the support of $\varphi$ does not intersect the hyperplane at infinity, then (3.10) is an entire function which vanishes at $\lambda=0$ ). We may suppose that the local coordinates in $V$ are $\xi:=\left(\frac{\zeta_{0}}{\zeta_{1}}, \ldots, \frac{\zeta_{n}}{\zeta_{1}}\right)$ (for example). Let

$$
f_{j}(\zeta)=\frac{\mathcal{P}_{j}(\widetilde{\zeta}) \zeta_{0}^{\delta_{j}+M-D_{j}}}{\zeta_{1}^{M+\delta_{j}}}, \quad j=1, \ldots, n
$$

expressed in the local coordinates $\xi$. Let us introduce a resolution of singularities $(\mathcal{X}, \pi)$ for the hypersurface $\left\{f_{1} \cdots f_{n}=0\right\}$ over $V$ (shrinking $V$ about the point $x$ if necessary). Then, in a local chart $\omega$ on $\mathcal{X}$ with coordinates $w$ centered at the origin, all functions $\pi^{*}\left(f_{j}\right)$ are, up to invertible holomorphic functions, monomials in $w$; that is

$$
\pi^{*}\left(f_{j}\right)(w)=u_{j}(w) w_{1}^{\theta_{j 1}} \cdots w_{n}^{\theta_{j n}}, \quad \theta_{j k} \in \mathbb{N}, u_{j} \text { invertible in } \omega
$$

Note that

$$
\pi^{*}\left[\frac{\zeta_{0}}{\zeta_{1}}\right](w)=u_{0}(w) w_{1}^{\theta_{01}} \cdots w_{n}^{\theta_{0 n}}, \quad \theta_{0 k} \in \mathbb{N}, u_{0} \text { invertible in } \omega
$$

since $\delta_{j}+M-D_{j}>0$ for at least one $j$ (in fact for any $j$ ). However this is not enough. Using the ideas of Varchenko [30] and Khovanskii [23], we introduce, above each such local chart $\omega$, a toroidal manifold $\widetilde{\mathcal{X}}$ and a proper holomorphic map $\widetilde{\pi}: \widetilde{\mathcal{X}} \mapsto \omega$ (which is locally a biholomorphism between $\widetilde{\mathcal{X}} \backslash \widetilde{\pi}^{*}\left\{w_{1} \cdots w_{n}=0\right\}$ and $\left.\omega \backslash\left\{w_{1} \cdots w_{n}=0\right\}\right)$, such that, on each local chart $\widetilde{\omega}$ on $\widetilde{\mathcal{X}}$ (with local coordinates $\left(t_{1}, \ldots, t_{n}\right)$ ), one has

$$
\tilde{\pi}^{*} \pi^{*}\left(f_{j}\right)\left(t_{1}, \ldots, t_{n}\right)=\widetilde{u}_{j}(t) t_{1}^{\widetilde{\theta}_{j 1}} \cdots t_{n}^{\widetilde{\theta}_{j n}}=\widetilde{u}_{j}(t) \widetilde{m}_{j}(t)
$$

and one of the $\widetilde{m}_{j}, j=1, \ldots, n$, let say $\widetilde{m}$, divides $\widetilde{m}_{1}, \ldots, \widetilde{m}_{n}$. Namely, the manifold $\widetilde{\mathcal{X}}$ is the smooth toric variety attached to a simple refinement of the fan associated with the Newton polyhedron

$$
\Gamma^{+}\left(\theta_{1}, \ldots, \theta_{n}\right):=\bigcup_{j=1}^{n}\left[\theta_{j}+\mathbb{N}^{n}\right] .
$$

It arises from gluing together copies $\left(U_{J}, \pi_{J}\right)$ of $\mathbb{C}^{n}$ (in correspondence with the $n$-dimensional cones of the fan, $\pi_{J}$ being a monoidal transform attached to the skeleton of the cone), according to the gluing of the cones along their edges. The 1-dimensional edges of these cones are determined as the normal directions to the $(n-1)$-dimensional faces of the Newton polyhedron $\Gamma^{+}\left(\theta_{1}, \ldots, \theta_{n}\right)$, plus a minimal system of additional rational directions in $\left[0, \infty\left[{ }^{n}\right.\right.$ (which are just necessary for the fan to be simple). 
We now come to the crucial point where we use the hypothesis (1.7), which tells us that, for $R\left|\zeta_{0}\right| \leqslant\left(\left|\zeta_{1}\right|^{2}+\cdots+\left|\zeta_{n}\right|^{2}\right)^{/ 12}$, one has

$$
\left|\zeta_{0}\right|^{M}\|\widetilde{\zeta}\|^{|\delta|} \leqslant c \sum_{j=1}^{n}\left|\mathcal{P}_{j}(\widetilde{\zeta})\left\|\left.\zeta_{0}\right|^{M+\delta_{j}-D_{j}}\right\| \widetilde{\zeta}\left\|^{\delta_{[j]}} \leqslant c_{n}\right\| \mathcal{P} \|_{\delta, M}\right.
$$

This implies that, if

$$
\widetilde{\pi}^{*} \pi^{*}\left[\zeta_{0} \zeta_{1}\right](t)=\widetilde{u}_{0}(t) t_{1}^{\widetilde{\theta}_{01}} \cdots t_{n}^{\widetilde{\theta}_{0 n}}, \quad \widetilde{u}_{0} \text { invertible in } \widetilde{\omega}
$$

the distinguished monomial $\widetilde{m}$ divides $\widetilde{m}_{0}^{M}$, where $\widetilde{m}_{0}:=t_{1}^{\widetilde{\theta}_{01}} \cdots t_{n}^{\widetilde{\theta}_{0 n}}$, in $\widetilde{\omega}$. Let $\widetilde{\varphi}$ be a test function on $\mathcal{X}$ with support in the local chart $\omega$. As can be easily seen, one can write in $\widetilde{\omega}$,

$$
\widetilde{\pi}^{*}\left[\widetilde{\varphi}\left[\pi^{*}\left(\varphi A_{P, Q}\left(\frac{\zeta_{1}}{\zeta_{0}}, \ldots, \frac{\zeta_{n}}{\zeta_{0}}\right)\right)\right]\right](t)=\frac{\widetilde{m}_{0}^{n M+|\delta|}}{\widetilde{m}^{n}}\left(\frac{\overline{\partial \widetilde{m}}}{\overline{\widetilde{m}}} \wedge \sigma_{1}(t)+\tau_{1}(t)\right),
$$

where $\sigma_{1}$ and $\tau_{1}$ (depending on $\varphi$ and $\widetilde{\varphi}$ ) are smooth differential forms with respective type $(n, n-1)$ and $(n, n)$. It follows then from (3.8) that

$$
\widetilde{\pi}^{*}\left[\widetilde{\varphi}\left[\pi^{*}\left(\varphi \bar{\partial} \Theta_{P, Q, \lambda}^{\delta, M}\right)\right]\right](t)=\lambda|\widetilde{m}|^{2 \lambda}|\xi|^{2 \lambda} \frac{\widetilde{m}_{0}^{n M+|\delta|-\operatorname{deg} Q-n-1}}{\widetilde{m}^{n}}\left(\frac{\overline{\partial \widetilde{m}}}{\overline{\widetilde{m}}} \wedge \sigma_{2}(t)+\tau_{2}(t)\right),
$$

where $\sigma_{2}$ and $\tau_{2}$ (depending on $\varphi$ and $\widetilde{\varphi}$ ) are smooth differential forms with respective type $(n, n-1)$ and $(n, n)$ and $\xi$ is a real analytic strictly positive function in $\widetilde{\omega}$. Since $|\delta|-\operatorname{deg} Q-$ $n-1 \geqslant 0$ and $\widetilde{m}^{n}$ divides $\widetilde{m}_{0}^{n M}$, we get immediately that for any test function $\rho$ with support in $\widetilde{\omega}$

$$
\left[\int \rho(t) \widetilde{\pi}^{*}\left[\widetilde{\varphi}\left[\pi^{*}\left(\bar{\partial} \Theta_{P, Q, \lambda}^{\delta, M}\right)\right]\right](t)\right]_{\lambda=0}=0 .
$$

Then, the conclusion (1.8) follows from the formula (3.9) and our localization and normalized blowing-up process.

Remark 3.1. - The fact that $\delta_{j}>0$ does not play any role in the proof. Therefore, Theorem 1.1 remains valid when $\left(P_{1}, \ldots, P_{n}\right)$ is $\left(\delta_{1}, \ldots, \delta_{n}\right)$-proper, where the $\delta_{j}$ are rational numbers such that $\delta_{j} \leqslant D_{j}$ for any $j=1, \ldots, n$ (see Remark 2.1); of course, the conclusion of the theorem is interesting only in the case when $\delta_{1}+\cdots+\delta_{n} \geqslant n+1$.

\subsection{The toric case}

We begin with a review of some preliminary material taken from $[9-12,17]$.

A complete toric variety $\mathcal{X}$ of dimension $n$ is determined by a complete fan $\mathcal{F}$ in an $n$ dimensional real vector space $\Lambda_{\mathbb{R}}$, where $\Lambda$ is a lattice; for the sake of simplicity, we will always assume $\Lambda=\mathbb{Z}^{n}$ and $\Lambda_{\mathbb{R}}=\mathbb{R}^{n}$. Taking a suitable refinement of the fan, we may assume that this toric variety $\mathcal{X}$ is also smooth.

We denote as $\Lambda^{*} \simeq \mathbb{Z}^{n}$ the dual lattice. The primitive generators of the one-dimensional cones in $\mathcal{F}$ are denoted by $\eta_{1}, \ldots, \eta_{s}$. Each of these vectors $\eta_{i}, i=1, \ldots, s$, is in correspondence with a torus-invariant irreducible Weil divisor $\mathcal{X}_{i}$ on $\mathcal{X}$. The $(n-1)$-Chow group $\mathbb{A}_{n-1}(\mathcal{X})$ on $\mathcal{X}$ is generated by the classes $\left[\mathcal{X}_{i}\right], i=1, \ldots, s$, and induces a grading on the polynomial algebra 
$\mathbb{S}=\mathbb{C}\left[x_{1}, \ldots, x_{s}\right]$, namely

$$
\operatorname{deg}\left(x_{1}^{\alpha_{1}} \cdots x_{s}^{\alpha_{s}}\right):=\left[\alpha_{1} \mathcal{X}_{1}+\cdots+\alpha_{s} \mathcal{X}_{s}\right] \in \mathbb{A}_{n-1}(\mathcal{X}) .
$$

Note that the sequence

$$
0 \longrightarrow \Lambda^{*} \stackrel{\tau}{\longrightarrow} \mathbb{Z}^{s} \longrightarrow \mathbb{A}_{n-1}(\mathcal{X}) \longrightarrow 0
$$

where $\tau(m)=\left(\left\langle m, \eta_{1}\right\rangle, \ldots,\left\langle m, \eta_{s}\right\rangle\right) \in \mathbb{Z}^{s}$ is exact since any monomial $x^{\langle m, \eta\rangle}:=x_{1}^{\left\langle m, \eta_{1}\right\rangle} \ldots$ $x_{s}^{\left\langle m, \eta_{s}\right\rangle}, m \in \Lambda^{*}$, has degree zero. If $\left(e_{1}^{*}, \ldots, e_{n}^{*}\right)$ is the canonical basis of $\Lambda^{*}$ and $\mathcal{I}$ is an ordered subset of $\{1, \ldots, s\}$ with cardinal $|\mathcal{I}|=n$, let say $\mathcal{I}=\left\{i_{1}, \ldots, i_{n}\right\}, 1 \leqslant i_{1}<\cdots<i_{n} \leqslant s$, we denote as

$$
\mathrm{d} x_{\mathcal{I}}:=\bigwedge_{\ell=1}^{n} \mathrm{~d} x_{i_{\ell}}, \quad \widehat{x_{\mathcal{I}}}:=\prod_{\substack{k=1 \\ k \notin \mathcal{I}}}^{s} x_{k}, \quad \operatorname{det}\left[\eta_{\mathcal{I}}\right]:=\operatorname{det}\left[\left\langle e_{k}^{*}, \eta_{i_{\ell}}\right\rangle\right]_{1 \leqslant k, \ell \leqslant n} .
$$

The toric Euler form on $\mathcal{X}$ is the differential form $\Omega$ (expressed in homogeneous coordinates $\left.x_{1}, \ldots, x_{s}\right)$

$$
\Omega(x):= \pm 1 \sum_{|\mathcal{I}|=n} \operatorname{det}\left[\eta_{\mathcal{I}}\right] \widehat{x_{\mathcal{I}}} \mathrm{d} x_{\mathcal{I}}
$$

We now consider a system $\left(F_{1}, \ldots, F_{n}\right)$ of Laurent polynomials with respective polyhedra $\Delta_{1}, \ldots, \Delta_{n}$, and a collection $\left(\delta_{1}, \ldots, \delta_{n}\right)$ of rational polyhedra such that $\delta_{j} \subset \Delta_{j}$ for any $j \in\{1, \ldots, n\}, \delta_{1}+\cdots+\delta_{n}$ is $n$-dimensional and the hypothesis (1.9) are fulfilled.

Before proceeding any further, by using the same change of basis (namely replace $X_{j}$ by $X_{j}^{N}$ for a convenient choice of $N \in \mathbb{N}^{*}$ ) as we did in (3.5), we can reduce ourselves to the situation where all polyhedra $\delta_{1}, \ldots, \delta_{n}$ have their vertices in the lattice $\Lambda=\mathbb{Z}^{n}$ (originally these vertices were assumed to be in $\mathbb{Q}^{n}$, therefore it is enough to take for $N$ a common denominator of all coordinates of such points).

We fix a polyhedron $\Delta$ with dimension $n$ and vertices in $\Lambda$, which contains the origin as an interior point and is such that, for any $j \in\{1, \ldots, n\}$, the Minkowski sum $\Delta+\delta_{j}$ contains $\Delta_{j}$. We let

$$
\widetilde{\Delta}:=\left(\Delta+\delta_{1}+\cdots+\delta_{n}\right)+\Delta_{1}+\cdots+\Delta_{n} .
$$

We consider as the fan $\mathcal{F}$ a simple refinement of the fan $\mathcal{F}(\widetilde{\Delta})$ which corresponds to this polyhedron $\widetilde{\Delta}$ (see [17]); $\mathcal{X}$ will be from now on the toric variety attached to $\mathcal{F}$. It is compatible with $\Delta, \delta_{j}+\Delta$ and $\Delta_{j}$ for any $j$. For any $j=1, \ldots, n$, we take $n+1$ Laurent polynomials, with convex polyhedron $\Delta+\delta_{j}$, namely $G_{0}^{(j)}, \ldots, G_{n}^{(j)}$, which do not vanish simultaneously in $\mathbb{T}^{n}$ and are such that the system $\left(G_{1}^{(j)}, \ldots, G_{n}^{(j)}\right)$ satisfies the Bernstein conditions (1.3) (when considered as a system of Laurent polynomials with Newton polyhedron $\Delta+\delta_{j}$ ). Since the fan $\mathcal{F}$ is compatible with $\Delta+\delta_{j}$, these Laurent polynomials induce Cartier divisors $\mathcal{D}_{0}^{(j)}, \ldots, \mathcal{D}_{n}^{(j)}$ on $\mathcal{X}$ such that

$$
\left|\mathcal{D}_{0}^{(j)}\right| \cap \cdots \cap\left|\mathcal{D}_{n}^{(j)}\right|=\emptyset .
$$

In particular, the function

$$
\left\|G^{(j)}\right\|^{2}:=\sum_{k=0}^{n}\left|G_{k}^{(j)}\right|^{2}
$$


does not vanish on the torus $\mathbb{T}^{n}$. Let, for $\zeta \in \mathbb{T}^{n}$,

$$
\begin{gathered}
\|F(\zeta)\|_{\delta, \Delta}^{2}:=\sum_{j=1}^{n} \frac{\left|F_{j}(\zeta)\right|^{2}}{\left\|G^{(j)}(\zeta)\right\|^{2}} \\
s_{0}^{\delta, \Delta}(\zeta):=\left(\frac{\overline{F_{1}(\zeta)}}{\left\|G^{(1)}(\zeta)\right\|^{2}}, \ldots, \frac{\overline{F_{n}(\zeta)}}{\left\|G^{(n)}(\zeta)\right\|^{2}}\right)
\end{gathered}
$$

and, for $\zeta \in \mathbb{T}^{n} \backslash V^{*}(F)$,

$$
s^{\delta, \Delta}(\zeta):=\frac{s_{0}^{\delta, \Delta}(\zeta)}{\|F(\zeta)\|_{\delta, \Delta}^{2}} .
$$

Let $\varepsilon=\min \left\{\left\|\zeta-\zeta^{\prime}\right\| ; \zeta \neq \zeta^{\prime}, \zeta, \zeta^{\prime} \in V^{*}(F)\right\}$ and

$$
U:=\bigcup_{\alpha \in V^{*}(F)} B\left[\alpha, \frac{\min _{\alpha \in V^{*}(F)}\left(\varepsilon, \mathrm{d}\left(\alpha, \mathbb{C}^{n} \backslash \mathbb{T}^{n}\right)\right)}{2}\right],
$$

where $d$ is the Euclidean distance in $\mathbb{C}^{n}$.

At this point, we notice that the representations (3.3) and (3.4) of the Bochner-Martinelli type that we gave to express the total sum of residues respect to a $n$-valued polynomial map from $\mathbb{C}^{n}$ to $\mathbb{C}^{n}$ remain valid when polynomials are replaced by Laurent polynomials $F_{j}, j=1, \ldots, n$, and total sum of residues involves only residues at poles in the torus $\mathbb{T}^{n}$, provided the $F_{j}, j=$ $1, \ldots, n$, define a discrete (hence finite) zero set in $\mathbb{T}^{n}$. Therefore, we may state the exact analog of Lemma 3.1 (the proof is a repetition of the proof of this previous lemma):

LEMMA 3.2. - Let $F=\left(F_{1}, \ldots, F_{n}\right)$ be a system of Laurent polynomials in $n$ variables, with respective Newton polyhedra $\Delta_{1}, \ldots, \Delta_{n}$ and polyhedra $\delta_{1}, \ldots, \delta_{n}, \Delta$ as above. Then, for any $\beta \in \mathbb{Z}$, one has

$$
\begin{aligned}
& \operatorname{Res}\left[\begin{array}{c}
X_{1}^{\beta_{1}} \cdots X_{n}^{\beta_{n}} \mathrm{~d} X \\
F_{1}, \ldots, F_{n}
\end{array}\right]_{\mathbb{T}} \\
& =\gamma_{n}\left[\int_{\partial U}\|F\|_{\delta, \Delta}^{2(\lambda-n)} \zeta^{\beta}\left(\sum_{k=1}^{n}(-1)^{k-1} s_{0 k}^{\delta, \Delta} \mathrm{d} s_{0,[k]}^{\delta, \Delta}\right) \wedge \frac{\mathrm{d} \zeta_{1}}{\zeta_{1}} \wedge \cdots \wedge \frac{\mathrm{d} \zeta_{n}}{\zeta_{n}}\right]_{\lambda=0} .
\end{aligned}
$$

We now adapt the construction (and study) of the meromorphic map

$$
\lambda \longmapsto \Theta_{P, Q, \lambda}^{\delta, M}
$$

that was proposed in (3.7). Such a meromorphic map will be defined in this new situation as follows: for $\lambda$ fixed with $\operatorname{Re} \lambda \gg 1$, let

$$
\Theta_{F, \beta, \lambda}^{\delta, \Delta}:=\|F\|_{\delta, \Delta}^{2(\lambda-n)} \zeta^{\beta}\left(\sum_{k=1}^{n}(-1)^{k-1} s_{0 k}^{\delta, \Delta} \mathrm{d} s_{0,[k]}^{\delta, \Delta}\right) \wedge \mathrm{d} \zeta
$$

(obtained from the integrant in (3.11) exactly as (3.7) was obtained from the integrant in (3.6)). As we did previously (in the projective situation), we express it in homogeneous coordinates $\left(x_{1}, \ldots, x_{s}\right)$ related to the toric variety $\mathcal{X}$. The coordinates $\zeta_{j}, j=1, \ldots, n$, in the torus are 
expressed in homogeneous coordinates as

$$
\zeta_{j}=\prod_{i=1}^{s} x_{i}^{\eta_{i j}}:=x^{\left\langle e_{j}^{*}, \eta\right\rangle}, \quad j=1, \ldots, n
$$

where, for any $i=1, \ldots, s, \eta_{i j}, j=1, \ldots, n$, are the coordinates of the primitive vector $\eta_{i}$ in the canonical basis $\left(e_{1}, \ldots, e_{n}\right)$ of $\Lambda \simeq \mathbb{Z}^{n}$. We will also define, for any $i \in\{1, \ldots, s\}$ and any $j \in\{1, \ldots, n\}$

$$
\left\{\begin{array}{l}
\mu_{i j}:=-\min _{\xi \in \Delta_{j}}\left\langle\xi, \eta_{i}\right\rangle, \\
\nu_{i j}:=-\min _{\xi \in \delta_{j}+\Delta}\left\langle\xi, \eta_{i}\right\rangle .
\end{array}\right.
$$

Since the situation is a little bit more involved than it was in the projective case, we will state the result of this homogenization process (applied to the differential form (3.12) and its $\bar{\partial}$ with respect to the $\zeta$ coordinates) as a lemma.

LEMMA 3.3. - The differential (n, $n-1)$-form in (3.12) can be expressed in homogeneous coordinates as

$$
\begin{aligned}
& \Theta_{F, \beta, \lambda}^{\delta, \Delta}(x)=\|\mathcal{F}(x)\|_{\delta, \Delta}^{2(\lambda-n)} \prod_{i=1}^{s} x_{i}^{\left\langle\beta, \eta_{i}\right\rangle-1+\sum_{j=1}^{n} \nu_{i j}} \\
& (3.15) \times\left(\sum_{k=1}^{n}(-1)^{k-1} \frac{\left[\left(\prod_{i=1}^{s} \overline{x_{i}^{\nu_{i k}-\mu_{i k}}}\right) \overline{\mathcal{F}_{j}(x)}\right]}{\left\|\mathcal{G}^{(k)}(x)\right\|^{2}} \bigwedge_{\substack{\ell=1 \\
\ell \neq k}}^{n} \bar{\partial}\left[\frac{\left(\prod_{i=1}^{s} \overline{x_{i}^{\nu_{i \ell}-\mu_{i \ell}}}\right) \overline{\mathcal{F}_{\ell}(x)}}{\left\|\mathcal{G}^{(\ell)}(x)\right\|^{2}}\right]\right) \wedge \Omega(x) .
\end{aligned}
$$

The differential form $\bar{\partial}_{\zeta} \Theta_{F, \beta, \lambda}^{\delta, \Delta}$ can be expressed as

$$
\begin{aligned}
& \bar{\partial} \Theta_{F, \beta, \lambda}^{\delta, \Delta}(x) \\
& \quad=n \lambda\|\mathcal{F}(x)\|_{\delta, \Delta}^{2(\lambda-n)}\left(\prod_{i=1}^{s} x_{i}^{\left\langle\beta, \eta_{i}\right\rangle-1+\sum_{j=1}^{n} \nu_{i j}}\right) \bigwedge_{k=1}^{n} \bar{\partial}\left[\frac{\left(\prod_{i=1}^{s} \overline{x_{i}^{\nu_{i k}-\mu_{i k}}}\right) \overline{\mathcal{F}_{k}(x)}}{\left\|\mathcal{G}^{(k)}(x)\right\|^{2}}\right] \wedge \Omega(x) .
\end{aligned}
$$

This means that the differential forms on $\mathcal{X}$ which are such defined in homogeneous coordinates restrict respectively to the torus as the differential forms $\Theta_{F, \beta, \lambda}^{\delta, \Delta}(\zeta)$ and its $\bar{\partial}$ in $\zeta$.

Proof. - Consider the $\mathcal{X}$-homogenizations of $F_{1}, \ldots, F_{n}$, that is

$$
\mathcal{F}_{j}\left(x_{1}, \ldots, x_{s}\right):=\left(\prod_{i=1}^{s} x_{i}^{\mu_{i j}}\right) F_{j}\left(x^{\left\langle e_{1}^{*}, \eta\right\rangle}, \ldots, x^{\left\langle e_{n}^{*}, \eta\right\rangle}\right),
$$

and the $\mathcal{X}$-homogenizations of the $G_{k}^{(j)}, j=1, \ldots, n, k=0, \ldots, n$, namely

$$
\mathcal{G}_{k}^{(j)}\left(x_{1}, \ldots, x_{s}\right):=\left(\prod_{i=1}^{s} x_{i}^{\nu_{i j}}\right) G_{k}^{(j)}\left(x^{\left\langle e_{1}^{*}, \eta\right\rangle}, \ldots, x^{\left\langle e_{n}^{*}, \eta\right\rangle}\right) .
$$


We will also denote

$$
\left\|\mathcal{G}^{(j)}\left(x_{1}, \ldots, x_{s}\right)\right\|^{2}:=\sum_{k=0}^{n}\left|\mathcal{G}_{k}^{(j)}(x)\right|^{2}, \quad j=1, \ldots, n .
$$

The function

$$
\zeta \longmapsto\|F(\zeta)\|_{\delta, \Delta}^{2}
$$

on the torus will be extended as the function on $\mathcal{X}$ which is defined in homogeneous coordinates as

$$
\|\mathcal{F}(x)\|_{\delta, \Delta}^{2}:=\sum_{j=1}^{n}\left|\prod_{i=1}^{s} x_{i}^{\nu_{i j}-\mu_{i j}}\right|^{2} \frac{\left|\mathcal{F}_{j}(x)\right|^{2}}{\left\|\mathcal{G}^{(j)}(x)\right\|^{2}} .
$$

On the other hand, one has, for $k=1, \ldots, n$,

$$
s_{0 k}^{\delta, \Delta}\left(x^{\left\langle e_{1}^{*}, \eta\right\rangle}, \ldots, x^{\left\langle e_{n}^{*}, \eta\right\rangle}\right)=\left|\prod_{i=1}^{s} x_{i}^{\nu_{i k}}\right|^{2} \frac{\left(\prod_{i=1}^{s} \overline{x_{i}^{-\mu_{i k}}}\right) \overline{\mathcal{F}_{k}(x)}}{\left\|\mathcal{G}^{(k)}(x)\right\|^{2}},
$$

while the differential form

$$
\left(\prod_{i=1}^{s} x_{i}^{\left\langle\beta, \eta_{i}\right\rangle}\right) \frac{\Omega(x)}{x_{1} \cdots x_{s}}
$$

on $\mathcal{X}$ restricts to the torus as $\zeta^{\beta} \frac{\mathrm{d} \zeta_{1}}{\zeta_{1}} \wedge \cdots \wedge \frac{\mathrm{d} \zeta_{n}}{\zeta_{n}}$ (see the proof of Proposition 9.5 in [2]). When we report these expressions in (3.12), we obtain the desired relations (3.15); the same kind of argument holds for (3.16).

The next lemma will be crucial in order to study in local charts about any point in $\mathcal{X} \backslash \mathbb{T}^{n}$ (under the "properness" assumption (1.9) for the $F_{j}$ ) the Laurent development (as current-valued maps) of

$$
\lambda \longmapsto \Theta_{F, \beta, \lambda}^{\delta, \Delta}, \quad \lambda \longmapsto \bar{\partial}_{\zeta}\left[\Theta_{F, \beta, \lambda}^{\delta, \Delta}\right]
$$

when such maps are expressed in homogeneous coordinates.

LEMMA 3.4. - Let $F=\left(F_{1}, \ldots, F_{n}\right)$ be a system of Laurent polynomials in $n$ variables, with respective Newton polyhedra $\Delta_{1}, \ldots, \Delta_{n}$. Suppose there exist constants $c>0, R>0$, and convex polyhedra $\delta_{1}, \ldots, \delta_{n}$ with vertices in $\mathbb{Z}^{n}$, with $\delta_{j} \subset \Delta_{j}, j=1, \ldots, n$ and $\operatorname{dim}\left(\delta_{1}+\right.$ $\left.\cdots+\delta_{n}\right)=n$, which are such that the hypothesis (1.9) is fulfilled. Let $\Delta$ be any polyedron with dimension $n$, vertices in $\mathbb{Z}^{n}$, such that $\Delta_{j} \subset \Delta+\delta_{j}$ for any $j=1, \ldots, n$ and $\mathcal{F}$ be a fan which is compatible with $\Delta, \delta_{j}+\Delta, \Delta_{j}$ for any $j=1, \ldots, n$. Let $\eta_{1}, \ldots, \eta_{s}$ be the primitive generators of the one-dimensional cones in the fan $\mathcal{F}$. Then, for any $\beta$ in $\mathbb{Z}^{n}$ which lies in the interior of the convex polyhedron $\delta_{1}+\cdots+\delta_{n}$, the integers $\nu_{i, j}$ defined in (3.14) are such that for any $i \in\{1, \ldots, s\}$, for any $j \in\{1, \ldots, n\}$

$$
\left\langle\beta, \eta_{i}\right\rangle-1+\sum_{j=1}^{n} \nu_{i j} \geqslant 0 .
$$


Remark 3.2. - Notice that the integers $\mu_{i, j}, i=1, \ldots, s, j=1, \ldots, n$, also defined in (3.14) satisfy automatically

$$
\nu_{i, j}-\mu_{i, j} \geqslant 0, \quad \forall i \in\{1, \ldots, s\}, \forall j \in\{1, \ldots, n\}
$$

since $\Delta_{j}$ is contained in $\delta_{j}+\Delta$ for any $j=1, \ldots, n$.

Proof of Lemma 3.4. - This lemma comes from a re-interpretation of our hypothesis (1.9). Since the system of polynomials $G_{k}^{(j)}, k=1, \ldots, n$, satisfies the Bernstein hypothesis (1.3), it follows (see the argument used in the proof of Proposition 2.2) that there exist strictly positive constants $c_{j}, C_{j}$, such that

$$
\forall \zeta \in \mathbb{C}^{n}, \quad c_{j} \mathrm{e}^{H_{\Delta+\delta_{j}}(\operatorname{Re} \zeta)} \leqslant\left\|G^{(j)}\left(\mathrm{e}^{\zeta_{1}}, \ldots, \mathrm{e}^{\zeta_{n}}\right)\right\| \leqslant C_{j} \mathrm{e}^{H_{\Delta+\delta_{j}}(\operatorname{Re} \zeta)} .
$$

One has also, for any $\zeta \in \mathbb{C}^{n}$ such that $\|\operatorname{Re} \zeta\| \geqslant R$,

$$
\sum_{j=1}^{n} \frac{\left|F_{j}\left(\mathrm{e}^{\zeta_{1}}, \ldots, \mathrm{e}^{\zeta_{n}}\right)\right|}{\mathrm{e}^{H_{\delta_{j}}(\operatorname{Re} \zeta)}} \geqslant c>0
$$

Note furthermore that $H_{\delta_{j}+\Delta}=H_{\delta_{j}}+H_{\Delta}$. We also introduce $n+1$ Laurent polynomials $H_{0}, \ldots, H_{n}$, with Newton polyhedron $\Delta$, which do not vanish simultaneously in $\mathbb{T}^{n}$ and are such that the system $\left(H_{1}, \ldots, H_{n}\right)$ satisfies the Bernstein hypothesis (1.3) when considered as a system of Laurent polynomials with Newton polyhedron $\Delta$, that is such that

$$
c_{0} \mathrm{e}^{H_{\Delta}(\operatorname{Re} \zeta)} \leqslant\left\|H\left(\mathrm{e}^{\zeta_{1}}, \ldots, \mathrm{e}^{\zeta_{n}}\right)\right\| \leqslant C_{0} \mathrm{e}^{H_{\Delta}(\operatorname{Re} \zeta)}, \quad \zeta \in \mathbb{C}^{n}
$$

for some strictly positive constants $c_{0}, C_{0}$ (where $\|H\|^{2}:=\left|H_{0}\right|^{2}+\cdots+\left|H_{n}\right|^{2}$ ). It follows from (3.19), (3.20) and (3.21) that for any $\zeta \in \mathbb{T}^{n}$ such that

$$
\left|\zeta_{1}\right|+\cdots+\left|\zeta_{n}\right|+\frac{1}{\left|\zeta_{1}\right|}+\cdots+\frac{1}{\left|\zeta_{n}\right|}
$$

is large enough, one has

$$
\sum_{j=1}^{n} \frac{\left|F_{j}(\zeta)\right|}{\left\|G^{(j)}(\zeta)\right\|} \geqslant \frac{\widetilde{c}}{\|H(\zeta)\|}
$$

for some $\widetilde{c}>0$. If we express the $\zeta_{j}$ in terms of homogeneous coordinates on the toric variety $\mathcal{X}$ as in (3.13), we may rewrite (3.22) as

$$
\left|\prod_{i=1}^{s} x_{i}{ }^{-\min _{\xi \in \Delta}\left\langle\xi, \eta_{i}\right\rangle}\right| \leqslant \frac{1}{\widetilde{c}} \frac{1}{|\mathcal{H}(x)|}\left(\sum_{j=1}^{n}\left|\prod_{i=1}^{s} x_{i}^{\nu_{i j}-\mu_{i j}}\right| \frac{\left|\mathcal{F}_{j}(x)\right|}{\left\|\mathcal{G}^{(j)}(x)\right\|}\right),
$$

where $\|\mathcal{H}(x)\|:=\sum_{k=0}^{n} \mid \mathcal{H}_{j}(x) \|$, the $\mathcal{H}_{j}$ being defined as the $\mathcal{X}$-homogenizations of the $H_{j}$, namely

$$
\mathcal{H}_{j}\left(x_{1}, \ldots, x_{s}\right):=\left(\prod_{i=1}^{s} x_{i}{ }^{-\min _{\xi \in \Delta}\left\langle\xi, \eta_{i}\right\rangle}\right) H_{j}\left(x^{\left\langle e_{1}^{*}, \eta\right\rangle}, \ldots, x^{\left\langle e_{n}^{*}, \eta\right\rangle}\right)
$$


The fact that $\delta_{1}+\cdots+\delta_{n}$ is $n$-dimensional and that $\beta$ lies in the interior of this polyhedron implies that for $i=1, \ldots, s$, one has

$$
\left\langle\beta, \eta_{i}\right\rangle-1-\min _{\xi \in \delta_{1}+\cdots+\delta_{n}}\left\langle\xi, \eta_{i}\right\rangle=-\min _{\xi \in \delta_{1}+\cdots+\delta_{n}}\left\langle\xi-\beta, \eta_{i}\right\rangle-1 \geqslant 0
$$

and hence

$$
\begin{aligned}
\left\langle\beta, \eta_{i}\right\rangle-1+\sum_{j=1}^{n} \nu_{i j} & =\left\langle\beta, \eta_{i}\right\rangle-1-\sum_{j=1}^{n} \min _{\xi \in \Delta+\delta_{j}}\left\langle\xi, \eta_{i}\right\rangle \\
& =\left\langle\beta, \eta_{i}\right\rangle-1-\sum_{j=1}^{n} \min _{\substack{\xi \in \Delta \\
\xi_{j} \in \delta_{j}}}\left(\left\langle\xi+\xi_{j}, \eta_{i}\right\rangle\right. \\
& \geqslant-n \min _{\xi \in \Delta}\left\langle\xi, \eta_{i}\right\rangle \geqslant 0,
\end{aligned}
$$

since $\Delta$ contains the origin. This proves the inequalities (3.17).

At this point we may translate (in the spirit of [13]) in more geometric terms the statement in Lemma 3.4.

Proposition 3.1. - Let $F_{1}, \ldots, F_{n}, \Delta_{1}, \ldots, \Delta_{n}, \Delta$ and $\mathcal{F}$ as in Lemma 3.4, such that the toric variety $\mathcal{X}(\mathcal{F})$ is an n-dimensional complex manifold. Let $E(\Delta), E\left(\Delta+\delta_{1}\right), \ldots$, $E\left(\Delta+\delta_{n}\right)$, be the $\mathcal{T}$-Cartier divisors corresponding to $\Delta, \Delta+\delta_{1}, \ldots, \Delta+\delta_{n}$ and

$$
\widetilde{\mathcal{D}}_{j}:=\operatorname{div}\left(F_{j}\right)+E\left(\Delta+\delta_{j}\right), \quad j=1, \ldots, n .
$$

Let $x$ be a point in $\mathcal{X}(\mathcal{F}) \backslash \mathbb{T}^{n}$ which lies in the intersection of the supports of the $\widetilde{\mathcal{D}}_{j}$, $j=1, \ldots, n$ and $\left[E_{x}\right]$ be the exceptional divisor in the normalized blow-up $\pi: \mathcal{N}_{x} \mapsto V_{x}$ along the ideal sheaf generated in $\mathcal{O}_{V_{x}}$ by global sections $\widetilde{f}_{x 1}, \ldots, \widetilde{f}_{x n}$ for the divisors $\widetilde{\mathcal{D}}_{j}$ in some neighborhood $V_{x}$ of $x$. Then, if $f_{x}$ is a global section for $E(\Delta)$ in $V_{x}$, one has

$$
\left[\pi^{*} f_{x}\right] \geqslant\left[E_{x}\right]
$$

that is the order of vanishing of $\pi^{*} f_{x}$ on each irreducible component of $\left[E_{x}\right]$ exceeds the multiplicity of this component in the decomposition of $\left[E_{x}\right]$.

Proof. - It follows immediately from (3.23), such an inequality being extended from $\mathbb{T}^{n}$ to $V_{x}$, which is possible since $\mathbb{T}^{n}$ is dense in $\mathcal{X}$. Let

$$
\left[E_{x}\right]=\sum_{\ell} r_{x \ell} E_{x \ell}
$$

the $E_{x \ell}$ being the irreducible components and the $r_{x \ell}$ the associated multiplicities. Lifting (3.23) to the normalized blow-up $\mathcal{N}_{x}$, one can see that the order of vanishing $\rho_{x \ell}$ of $\pi^{*} f_{x}$ along any $E_{x \ell}$ is such that $\rho_{x \ell} \geqslant r_{x \ell}$ for any $\ell$, which is equivalent to the statement (3.24).

Proof of Theorem 1.2. - The proof of Theorem 1.2 follows exactly the same lines as the proof of our previous result Theorem 1.1. We consider the complement of $U$ in $\mathcal{X}$ as a $2 n$-chain $\Sigma$ in $\mathcal{X}$, and we deduce from Lemma 3.2 and from the definition of $\Theta_{F, \beta, \lambda}^{\delta, \Delta}$ that

$$
\operatorname{Res}\left[\begin{array}{c}
X_{1}^{\beta_{1}} \cdots X_{n}^{\beta_{n}} \mathrm{~d} X \\
F_{1}, \ldots, F_{n}
\end{array}\right]_{\mathbb{T}}=-\gamma_{n}\left[\int_{\partial \Sigma} \Theta_{F, \beta, \lambda}^{\delta, \Delta}\right]_{\lambda=0}=-\gamma_{n}\left[\int_{\Sigma} \bar{\partial}\left(\Theta_{F, \beta, \lambda}^{\delta, \Delta}\right)\right]_{\lambda=0}
$$


the notation []$_{\lambda=0}$ meaning that one takes the meromorphic continuation, and later on, the value at $\lambda=0$. In order to prove the vanishing of the residue symbol, it is enough to show that if $x$ is any point in $\mathcal{X} \backslash \mathbb{T}^{n}, V_{x}$ an arbitrary small neighborhood of $x$ in $\mathcal{X}$, and $\varphi \in \mathcal{D}\left(V_{x}\right)$, then the function

$$
\lambda \longmapsto \int_{V_{x}} \varphi \bar{\partial}\left(\Theta_{F, \beta, \lambda}^{\delta, \Delta}\right)
$$

can be continued as a meromorphic function of $\lambda$ which has no pole at $\lambda=0$ and vanishes at $\lambda=0$. In order to do that, we repeat the argument in the proof of Theorem 1.1 and use a resolution of singularities $\mathcal{Y} \stackrel{\pi}{\longrightarrow} V_{x}$, followed by toroidal resolutions $\widetilde{\mathcal{Y}}_{\omega} \stackrel{\widetilde{\pi}_{\omega}}{\longrightarrow} \omega$ over each local chart $\omega$ on $\mathcal{Y}$, such that in local coordinates $\left(t_{1}, \ldots, t_{n}\right)$ on a local chart $\varpi$ in some $\widetilde{\mathcal{Y}}_{\omega}$, one has

$$
\widetilde{\pi}_{\omega}^{*} \pi^{*} \widetilde{f}_{x j}=\widetilde{u}_{j}(t) t_{1}^{\widetilde{\theta}_{j 1}} \cdots t_{n}^{\widetilde{\theta}_{j n}}=\widetilde{u}_{j}(t) \widetilde{m}_{j}(t)
$$

and some of the $\widetilde{m}_{j}$, say $\widetilde{m}$, divides $\widetilde{m}_{1}, \ldots, \widetilde{m}_{n}$. For the same reasons that lead to (3.24) from the properness condition (1.9) when one was using a normalized blow-up instead of the tower of resolutions $\widetilde{\mathcal{Y}}_{\omega} \stackrel{\widetilde{\pi}_{\omega}}{\longrightarrow} \omega \stackrel{\pi}{\longrightarrow} V_{x}$, one can see that the properness condition implies that $\widetilde{m}$ divides $\widetilde{\pi}_{\omega}^{*} \pi^{*} f_{x}$ in the local chart $\varpi$. Therefore, it follows from Lemma 3.3 (formula (3.16)) and Lemma 3.4 (inequalities (3.17) and (3.18)) that for any test function $\widetilde{\varphi}$ on $\mathcal{Y}$ with support in $\omega$, one can write in $\varpi$,

$$
\tilde{\pi}_{\omega}^{*}\left[\widetilde{\varphi}\left[\pi^{*}\left(\varphi \bar{\partial} \Theta_{F, \beta, \lambda}^{\delta, M}\right)\right]\right](t)=\lambda|\widetilde{m}|^{2 \lambda}|\xi|^{2 \lambda}\left(\frac{\overline{\partial \widetilde{m}}}{\overline{\widetilde{m}}} \wedge \sigma(t)+\tau(t)\right),
$$

where $\sigma$ and $\tau$ (depending on $\varphi$ and $\widetilde{\varphi}$ ) are smooth differential forms with respective type $(n, n-1)$ and $(n, n)$ and $\xi$ is a real analytic strictly positive function in $\varpi$. Therefore,

$$
\lambda \longmapsto \widetilde{\pi}_{\omega}^{*}\left[\widetilde{\varphi}\left[\pi^{*}\left(\varphi \bar{\partial} \Theta_{F, \beta, \lambda}^{\delta, M}\right)\right]\right](t)
$$

can be continued as a distribution valued meromorphic map on $\varpi$, which has no pole at $\lambda=0$ and vanishes at this point. Since the meromorphic function (3.26) is expressed as a sum of functions of the form

$$
\lambda \int_{\varpi} \widetilde{\psi}_{\omega}^{*}\left[\widetilde{\varphi}\left[\pi^{*}\left(\bar{\partial} \Theta_{F, \beta, \lambda}^{\delta, M}\right)\right]\right](t)\left[\pi_{\omega}^{*} \pi \varphi\right](t),
$$

the vanishing of the residue symbol (3.26) follows. This concludes the proof of our Theorem 1.2.

\section{Some applications of vanishing theorems for global sums of residues}

The generalized Jacobi Theorems 1.1, 1.2 derived above have as a direct consequence the following nonstandard formulations of Cayley-Bacharach type theorems in the spirit of [18].

THEOREM 4.1.- Given a $\left(\delta_{1}, \ldots, \delta_{n}\right)$-proper polynomial mapping $P: \mathbb{C}^{n} \rightarrow \mathbb{C}^{n}$ with only simple zeroes $\mathcal{Z}=\left\{\alpha_{1}, \ldots, \alpha_{m}\right\}$ and an algebraic hypersurface $Y \subset \mathbb{C}^{n}$ of degree strictly smaller than $\delta_{1}+\cdots+\delta_{n}-n$, then if $Y$ contains all but one points $\alpha_{j}$, it necessarily also contains the last one. 
Proof. - Suppose $Y=\{Q=0\}$ and that the degree of $Q$ is at most $\delta_{1}+\cdots+\delta_{n}-n-1$. Recall that

$$
\operatorname{Res}_{\alpha}\left[\begin{array}{c}
Q\left(\zeta_{1}, \ldots, \zeta_{n}\right) \mathrm{d} \zeta \\
P_{1}, \ldots, P_{n}
\end{array}\right]=\frac{Q(\alpha)}{\mathcal{J}_{P}(\alpha)},
$$

where $\mathcal{J}_{P}(\alpha)$ is the value of the Jacobian of the $\left(\delta_{1}, \ldots, \delta_{n}\right)$-proper mapping $P$ at the simple common zero $\alpha$. The hypothesis on the degree of the hypersurface implies (if one uses Theorem 1.1) that

$$
\operatorname{Res}\left[\begin{array}{c}
Q\left(X_{1}, \ldots, X_{n}\right) \mathrm{d} X \\
P_{1}, \ldots, P_{n}
\end{array}\right]=0 .
$$

Therefore, if $Q$ vanishes at all points in $\mathcal{Z}$ but one, it vanishes in fact at any point in $\mathcal{Z}$.

Example 4.1. - Let $P=\left(P_{1}, P_{2}, P_{3}\right)$ be the polynomial map from $\mathbb{C}^{3}$ to $\mathbb{C}^{3}$ defined as

$$
\begin{aligned}
& P_{1}(X)=X_{1} X_{2} X_{3}, \\
& P_{2}(X)=\left(X_{1}^{3}+1\right)\left(X_{2}^{3}+1\right)\left(X_{3}^{3}+1\right), \\
& P_{3}(X)=\left(X_{1}^{3}-1\right)\left(X_{2}^{3}-1\right)\left(X_{3}^{3}-1\right) .
\end{aligned}
$$

Such a map is $(1,3,3)$-proper; the 54 common zeroes of $\left(P_{1}, P_{2}, P_{3}\right)$ are all simple. Since $\delta_{1}+\delta_{2}+\delta_{3}-4=3$, any cubic (such as $\left\{X_{1}^{3}+X_{2}^{3}+X_{3}^{3}=0\right\}$ ) which passes through all but one the common zeroes $\alpha_{j}$ contains necessarily the remaining one.

We may also state a toric version of a Cayley-Bacharach theorem. We begin with the following

Definition 4.1. - A $\left(\delta_{1}, \ldots, \delta_{n}\right)$-proper system $F=\left(F_{1}, \ldots, F_{n}\right)$ of Laurent polynomials is called fully $\left(\delta_{1}, \ldots, \delta_{n}\right)$-proper if $\operatorname{dim}\left(\delta_{1}+\cdots+\delta_{n}\right)=n$.

THEOREM 4.2. - Consider a fully $\left(\delta_{1}, \ldots, \delta_{n}\right)$-proper system of Laurent polynomials $F=$ $\left(F_{1}, \ldots, F_{n}\right)$ with only simple zeroes $\alpha_{1}, \ldots, \alpha_{m}$ in $\mathbb{T}^{n}$, and a Laurent polynomial $Q$, whose Newton polyhedron lies in the interior of $\delta_{1}+\cdots+\delta_{n}$. Then if the hypersurface $\{Q=0\}$ contains all but one points $\alpha_{j}$ it necessarily also contains the last one.

Proof. - The proof is a direct application of the Theorem 1.2, exactly as our previous result follows from Theorem 1.1.

Example 4.2. - Let $F_{1}, F_{2}$ as in Example 2.2. We have noticed (see Example 2.2) that $\left(F_{1}-F_{2}, F_{1}\right)$ satisfies the Bernstein hypothesis (1.3). Therefore, in this case, the number of common zeroes of $F_{1}, F_{2}$ in the torus equals two times the mixed volume of $[-2,2]^{2}$ and $\delta$, where

$$
\delta:=\overline{\{\operatorname{conv}\{(-2,2),(2,2),(1,1),(1,-1)\}},
$$

that is 28 . If $Q$ is a Laurent polynomial with support in the interior of $[-2,2]+\delta$, such that $\left\{\zeta \in \mathbb{T}^{2} ; Q(\zeta)=0\right\}$ contains 27 points among the common zeroes of $F_{1}, F_{2}$ in the torus, it contains necessarily the remaining one.

Example 4.3. - We will choose here an example which is slightly more elaborate than Example 2.2 (in fact, in Example 2.2, $\left(F_{1}-F_{2}, F_{1}\right)$ satisfies the Bernstein condition (1.3), which leads back immediately to the situation where Khovanskii's result (1.4) applies). Let $F_{1}, F_{2}$ be the pair of Laurent polynomials in two variables, of the form

$$
\begin{aligned}
F_{j}(X, Y)= & \lambda_{j}\left(X_{1}^{2} X_{2}^{2}+X_{1}^{2} X_{2}^{-2}\right)+\alpha_{j} X_{1} X_{2}+\beta_{j} X_{1} X_{2}^{-1} \\
& +\gamma_{j} X_{1}^{-1} X_{2}+\delta_{j} X_{1}^{-1} X_{2}^{-1}+\left(X_{1}^{-2} X_{2}^{2}+X_{1}^{-2} X_{2}^{-2}\right),
\end{aligned}
$$


where $\alpha_{j}, \beta_{j}, \gamma_{j}, \delta_{j}, \lambda_{j}, j=1,2$, are generic complex coefficients. One can check easily $\left(F_{1}, F_{2}\right)$ is $(\widetilde{\delta}, \widetilde{\delta})$-proper, where $\widetilde{\delta}$ is now the polyedron defined as

$$
\widetilde{\delta}=\overline{\operatorname{conv}\{(-1,1),(0,4 / 3),(1,1),(1,-1),(0,-4 / 3),(-1,-1)\}} .
$$

The number of common zeroes $\alpha$ of $F_{1}$ and $F_{2}$ in $\mathbb{T}^{2}$ equals now 24 (note that, comparing to Example 2.2, 4 among the 28 common zeroes disappear "at infinity" in this new situation). Moreover, one has

$$
\widetilde{\delta}+\widetilde{\delta}=\overline{\operatorname{conv}\{(-2,-2),(-2,2),(0,8 / 3),(2,2),(2,-2),(0,-8 / 3)\}},
$$

so that the interior of $\widetilde{\delta}+\widetilde{\delta}$ contains $[-1,1] \times[-2,2]$. Therefore, any sextic

$$
\left\{Q\left(X_{1}, X_{2}\right)=0 ; Q \in \mathrm{C}\left[X_{1}, X_{2}\right], \operatorname{Supp} Q \subset[0,2] \times[0,4]\right\}
$$

which passes through 23 among the common zeroes $\alpha$ also contains the remaining one.

Finally, we can state an application of Theorem 1.1 (respectively Theorem 1.2) to some effective version of division problems with respect to proper quasi-regular maps. In the first case, this version is the key ingredient for a general explicit formulation to the algebraic Nullstellensatz $[5,6]$; we do not know yet if the same holds in the toric case for the Newton Nullstellensatz.

Proposition 4.1. - Let $P:=\left(P_{1}, \ldots, P_{n}\right)$ be a $\left(\delta_{1}, \ldots, \delta_{n}\right)$-proper polynomial map from $\mathbb{C}^{n}$ to $\mathbb{C}^{n}$, where $\delta_{j}>0$ for any $j$; suppose that $\operatorname{deg} P_{j}=D_{j}, j=1, \ldots, n$. Let $Q_{j k}, j, k=$ $1, \ldots, n$, be polynomials in $\left(X_{1}, \ldots, X_{n}, Y_{1}, \ldots, Y_{n}\right)$ such that $\operatorname{deg} Q_{j k} \leqslant D_{j}-1, j=1, \ldots, n$, and

$$
P_{j}(Y)-P_{j}(X)=\sum_{k=1}^{n} Q_{j k}(X, Y)\left(Y_{k}-X_{k}\right)
$$

Let

$$
\operatorname{det}\left[Q_{j k}(X, Y)\right]_{1 \leqslant j, k \leqslant n}=\sum_{\substack{\alpha, \beta \in \mathbb{N}^{n} \\|\alpha|+|\beta| \leqslant D_{1}+\cdots+D_{n}-n}} \gamma_{\alpha, \beta} X^{\alpha} Y^{\beta} .
$$

Then for any polynomial $Q$ with degree $D$, one has the following identity

$$
Q(Y)=\sum_{\substack{\alpha, \beta \in \mathbb{N}^{n} \\
|\alpha|+|\beta| \leqslant D_{1}+\cdots+D_{n}-n}} \sum_{\substack{\mu \in \mathbb{N}^{n} \\
\langle\mu+1, \delta\rangle \leqslant|\alpha|+D+n}} \gamma_{\alpha, \beta} \operatorname{Res}\left[\begin{array}{c}
Q(X) X^{\alpha} \mathrm{d} X \\
P_{1}^{\mu_{1}+1}, \ldots, P_{n}^{\mu_{n}+1}
\end{array}\right] Y^{\beta} P(Y)^{\mu},
$$

where we used the standard notations: $\zeta^{m}=\zeta_{1}^{m_{1}} \cdots \zeta_{n}^{m_{n}}$ for $\zeta \in \mathbb{C}^{n}$ and $m \in \mathbb{N}^{n},\left\langle m_{1}, m_{2}\right\rangle=$ $m_{11} m_{21}+\cdots+m_{1 n} m_{2 n}$ for $m_{1}, m_{2} \in \mathbb{N}^{n}, \underline{1}=(1, \ldots, 1)$ ( $n$ times).

Proof. - The proof follows from the Cauchy-Weil integral representation formula, exactly as in [5]; the analytic expansion of the Cauchy kernel that appears in this formula truncates thanks to Theorem 1.1.

COROLlary 4.1. - Let $P:=\left(P_{1}, \ldots, P_{n}\right)$ be a $\left(\delta_{1}, \ldots, \delta_{n}\right)$ - proper polynomial map from $\mathbb{C}^{n}$ to $\mathbb{C}^{n}$, where $\delta_{j}>0$ for any $j$; let $Q$ be in the ideal $I\left(P_{1}, \ldots, P_{n}\right)$; then, one can write a 
division formula for $Q$ respect to the ideal $I\left(P_{1}, \ldots, P_{n}\right)$ as

$$
Q(Y)=\sum_{\substack{\mu \in\left(\mathbb{N}^{n}\right)^{*}, \nu \in \mathbb{N}^{n} \\|\nu| \leqslant D_{1}+\cdots+D_{n}-n \\\langle\mu+1, \delta\rangle+|\nu| \leqslant D_{1}+\cdots+D_{n}+\operatorname{deg} Q}} \tilde{\gamma}_{\mu, \nu} Y^{\nu} P(Y)^{\mu} .
$$

Note that if $\delta_{j}=D_{j}$ (that is the $P_{j}$ do not have common zeroes at infinity), formula (4.2) becomes

$$
Q(Y)=\sum_{\substack{\mu \in\left(\mathbb{N}^{n}\right)^{*}, \nu \in \mathbb{N}^{n} \\\langle\mu, D\rangle+|\nu| \leqslant \operatorname{deg} Q}} \widetilde{\gamma}_{\mu, \nu} Y^{\nu} P(Y)^{\mu}
$$

which is not a surprise since the homogenization $\mathcal{Q}$ of $Q$ lies (in this case) in the homogeneous ideal generated by $\mathcal{P}_{1}, \ldots, \mathcal{P}_{n}$.

In the toric case, we need first a definition, that we recall from [8, p. 454].

Definition 4.2. - Let $\Delta$ be a closed convex polyhedron in $\mathbb{R}^{n} ; \Delta$ is called a good polyhedron if and only if

$$
\forall x \in \Delta, \quad\left\{y \in \mathbb{R}^{n} ;\left|y_{k}\right| \leqslant\left|x_{k}\right|, x_{k} y_{k} \geqslant 0, k=1, \ldots, n\right\} \subset \Delta .
$$

We can now state the toric pendant of Proposition 4.1.

PROPOSITION 4.2. - Let $\delta_{1}, \ldots, \delta_{n}$ be $n$ convex rational polyhedra in $\mathbb{R}^{n}$ with dimension $n$ which contain the origin as an interior point; let $F:=\left(F_{1}, \ldots, F_{n}\right)$ be a system of Laurent polynomials with good Newton polyhedra $\Delta_{1}, \ldots, \Delta_{n}$, such that $\delta_{j} \subset \Delta_{j}$ for any $j$ and $F$ is $\left(\delta_{1}, \ldots, \delta_{n}\right)$-proper. Then one can find Laurent polynomials $G_{j k}, j, k=1, \ldots, n$, in $\left(X_{1}, \ldots, X_{n}, Y_{1}, \ldots, Y_{n}\right)$, such that

$$
\operatorname{det}\left[G_{j k}(X, Y)\right]_{1 \leqslant j, k \leqslant n}=\sum_{\substack{\alpha, \beta \in \mathbb{Z}^{n} \\ \alpha+\beta \in \Delta_{1}+\cdots+\Delta_{n}}} \gamma_{\alpha, \beta} X^{\alpha} Y^{\beta}
$$

and

$$
F_{j}(Y)-F_{j}(X)=\sum_{k=1}^{n} G_{j k}(X, Y)\left(Y_{k}-X_{k}\right), X, Y \in \mathbb{T}^{n}, \quad j=1, \ldots, n .
$$

Moreover, for any Laurent polynomial $G$ with convex polyhedron $\Delta$, one has the following algebraic identity

$$
G(Y)=\sum_{\substack{\alpha, \beta \in \mathbb{Z}^{n} \cap\left(\Delta_{1}+\cdots+\Delta_{n}\right) \\
\alpha+\beta \in \Delta_{1}+\cdots+\Delta_{n}}} \sum_{\substack{\mu \in \mathbb{N}^{n} \\
\Delta+\alpha+\underline{1} \not \subset \operatorname{int}(\langle\mu+\underline{1}, \delta\rangle)}} \gamma_{\alpha, \beta} \operatorname{Res}\left[\begin{array}{c}
G(X) X^{\alpha+1} \mathrm{~d} X \\
F_{1}^{\mu_{1}+1}, \ldots, F_{n}^{\mu_{n}+1}
\end{array}\right]_{\mathbb{T}} Y^{\beta} F(Y)^{\mu},
$$

where $\langle m, \delta\rangle:=m_{1} \delta_{1}+\cdots+m_{n} \delta_{n}$ for any $m \in \mathbb{N}^{n}$.

Proof. - For the construction of the $G_{j k}$ under the hypothesis that all $\Delta_{j}$ are good, we refer to [8]. The fact that one can get the algebraic identity (4.3) is based on the use of Cauchy-Weil formula, as in the proof of Proposition 4.1; for more details see [32], Section 2. The development of the Cauchy kernel as a geometric progression truncates (as claimed in (4.3)) if one applies Theorem 1.2. 
COROLlary 4.2. - Let $\left(F_{1}, \ldots, F_{n}\right)$ be a $\left(\delta_{1}, \ldots, \delta_{n}\right)$-proper system of Laurent polynomials; suppose that all $\delta_{j}$ are $n$ dimensional and contain the origin as an interior point; denote as $\Delta_{j}$ the smallest good polyhedron containing the support of $F_{j}, j=1, \ldots, n$. Then, whenever $G$ is a Laurent polynomial with Newton polyhedron $\Delta$ that lies in the ideal generated by $F_{1}, \ldots, F_{n}$ in $\mathbb{C}\left[X_{1}, \ldots, X_{n}, X_{1}^{-1}, \ldots, X_{n}^{-1}\right]$, one can write a division formula for $G$ respect to $\left(F_{1}, \ldots, F_{n}\right)$ as

$$
G(Y)=\sum_{\substack{\mu \in\left(\mathbb{N}^{n}\right)^{*}, \nu \in \mathbb{Z}^{n} \cap\left(\Delta_{1}+\cdots+\Delta_{n}\right) \\ \Delta+\Delta_{1}+\cdots+\Delta_{n} \not \subset \operatorname{int}\langle\mu+\underline{1}, \delta\rangle+\beta-\underline{1}}} \widetilde{\gamma}_{\mu, \nu} Y^{\nu} F(Y)^{\mu} .
$$

\section{Acknowledgments}

We are indebted to the Institut culturel français (ICF) in Cyprus and the University of Cyprus for the financial support they provided during the preparation of this work.

\section{REFERENCES}

[1] AтіYAн M.F., Resolution of singularities and division of distributions, Commun. Pure. Appl. Math. 23 (1970) 145-150.

[2] Batyrev V., Cox D., On the Hodge structure of projective hypersurfaces in toric varieties, Duke J. Math. 75 (1994) 293-338.

[3] Bernstein D., The number of roots of a system of equations, Funct. Anal. Appl. 9 (2) (1975) 183185.

[4] BRIANÇON J., SKODA H., Sur la clôture intégrale d'un idéal de germes de fonctions holomorphes en un point de $\mathbb{C}^{n}$, C. R. Acad. Sci. Paris, Série I 278 (1974) 949-951.

[5] Berenstein C.A., Yger A., A formula of Jacobi and its consequences, Ann. Scient. École Norm. Sup. 24 (1991) 73-83.

[6] Berenstein C.A., Yger A., Residue calculus and effective Nullstellensatz, Amer. J. Math. 121 (1999) 723-796.

[7] Berenstein C.A., Yger A., Residues and effective Nullstellensatz, ERA Amer. Math. Soc. 2 (2) (1996) 82-91.

[8] Berenstein C.A., YGer A., Multidimensional residues and complexity problems, Mathematics and Computers in Simulation 42 (1996) 449-457.

[9] Cattani E., Cox D., Dickenstein A., Residues in toric varieties, Compositio Math. 108 (1997) 35-76.

[10] Cattani E., Dickenstein A., A global view of residues in the torus, J. Pure Appl. Algebra $117 \&$ 118 (1997) 119-144.

[11] Cox D., The homogeneous coordinate ring of a toric variety, J. Algebraic Geom. 4 (1995) 17-50.

[12] Cox D., Recent developments in toric geometry, Proc. Symp. Pure Math., Vol. 62, Part 2, Amer. Math. Soc., Providence, RI, 1997.

[13] Ein L., LaZARsfeld R., A geometric effective Nullstellensatz, Invent. Math. 137 (1999) 427-448.

[14] Eisenbud D., Green M., Harris J., Cayley-Bacharach theorems and conjectures, Bull. Amer. Math. Soc. (N.S.) 33 (1996) 295-324.

[15] ERTL S., HüBL R., The Jacobian formula for Laurent polynomials, Universitatis Iagellonicae Acta Mathematica XXXVII (1999) 51-67.

[16] Fabiano A., Pucci G., YGer A., Effective Nullstellensatz and geometric degree for zerodimensional ideals, Acta Arithmetica 78 (2) (1996) 165-187.

[17] Fulton W., Introduction to Toric Varieties, Princeton Univ. Press, 1993.

[18] Griffiths P., Harris J., Principles of Algebraic Geometry, Wiley Interscience, New York, 1978.

4e SÉRIE - TOME $34-2001-\mathrm{N}^{\circ} 1$ 
[19] JАСОві C.G.J., De relationibus, quae locum habere denent inter puncta intersectionis duarum curvarum vel trium superficierum algebraicarum dati ordinis, simul cum enodatione paradoxi algebraici, Gesammelte Werke, Band III, pp. 329-354.

[20] Kazarnovskit B.JA., On the zeroes of exponential sums, Soviet. Math. Doklady 23 (1981) 347-351.

[21] Kazarnovskit B.JA., Newton polyhedra and zeros of systems of exponential sums, Funct. Anal. Appl. 18 (4) (1984) 299-307.

[22] KhovanskiI A., Newton polyhedra and the Euler-Jacobi formula, Russian Math. Surveys 33 (1978) 237-238.

[23] Khovanskit A., Newton polyhedra and the toroidal varieties, Funct. Anal. Appl. (1978) 289-295.

[24] Kollár J., Sharp effective Nullstellensatz, J. Amer. Math. Soc. 1 (1988) 963-975.

[25] Kollár J., Effective Nullstellensatz for arbitrary ideals, J. Eur. Math. Soc. 1 (1999) 313-337.

[26] Lipman J., Residues and traces of differential forms via Hochschild homology, Contemp. Math., Vol. 61, Amer. Math. Soc., Providence, 1987.

[27] Pellé T., Identités de Bézout pour certains systèmes de sommes d'exponentielles, Ark. Math. 36 (1998) 131-162.

[28] Passare M., Tsikh A., Yger A., Residue currents of the Bochner-Martinelli type, Publicaciones Math. 44 (2000) 85-117.

[29] Tsikh A., Multidimensional residues and their applications, Transl. Amer. Math. Soc. 103 (1992).

[30] Varchenko A., Newton polyhedra and estimation of oscillating integrals, Funct. Anal. Appl. 10 (1976) 175-196.

[31] Yger A., Lectures at Croce di Magara, June 1998, notes, available on the site www. math. u-bordeaux. fr/ yger.

[32] YGER A., Résidus, courants résiduels et courants de Green, in: Norguet F., Ofman S., Szczeciniarz J.J. (Eds.), Géométrie Complexe, Actualités Scientifiques et Industrielles, 1438, Hermann, Paris, 1996.

(Manuscript received April 22, 1999; accepted, after revision, January 20, 2000.)

Alekos VIDRAS

Department of Mathematics and Statistics,

University of Cyprus,

Nicosia, Cyprus

Alain YGER

Laboratoire de mathématiques pures de Bordeaux,

Université Bordeaux-1,

351, cours de la Libération,

33405 Talence cedex, France 